\title{
Influence functions for a linear subspace method
}

\author{
Kuniyoshi Hayashi \\ Graduate School of Environmental and Life Science, Okayama University, Japan \\ CREST, Japan Science and Technology Agency, Japan
}

\begin{abstract}
A linear subspace method, which is one of discriminant methods, was proposed as a pattern recognition method and was studied. Because the method and its extensions do not encounter the situation of singular covariance matrix, we need not consider extensions such as generalized ridge discrimination, even when treating a high dimensional and sparse dataset. In addition, classifiers based on a multi-class discrimination method can function faster because of the simple decision procedure. Therefore, they have been widely used for face and speech recognition. However, it seems that sufficient studies have not been conducted about the statistical assessment of training data performance for classier in terms of prediction accuracy. In statistics, influence functions for statistical discriminant analysis were derived and the assessments for analysis result were performed. These studies indicate that influence functions are useful for detecting large influential observations for analysis results by using discrimination methods and they contribute to enhancing the performance of a target classifier.

In this paper, we propose the statistical diagnostics of a classifier on the basis of an influence function by using the linear subspace method. We first propose the discriminant score for the linear subspace method. Next, we derive the sample and empirical influence functions for the average of the discriminant scores to detect large influential observations for the misclassification rate. Finally, through a simulation study and a real data analysis, we detect the outliers in the training dataset using the derived influence function and develop a highly sophisticated classifier in the linear subspace method.
\end{abstract}

Keywords: CLAFIC; Cross-validation; Perturbation analysis; Single-case diagnostics 


\section{Introduction}

The demands for discrimination methods that classify unknown high dimensional observations have accelerated in many fields. Watanabe $[20,21]$ proposed a linear subspace method to perform discrimination for a high dimensional pattern vector. If a vector pattern has relatively large number of features, this method can efficiently solve a discriminant problem with a fast calculation time, even if the number of target classes is large. Therefore, this method has been used in real time recognition. The linear subspace method and its extensions have been studied by numerous authors, including Watanabe and Pakvasa [22], Kittler [12], Karhunen and Oja [10], Oja [16], Kohonen [13], Laaksonen and Oja [14], Maeda and Murase [15], Gülmezoğlu et al. [6], Gunal and Edizkan [5], Kitamura et al. [11], and Yamashita and Wakahara [23]. In the field of machine learning and pattern recognition, there are many powerful classifiers. In pattern recognition, the extensions of CLAFIC method have been widely used in face and speech recognition. For example, kernel nonlinear subspace method is an extended method of CLAIFC method and it was powerful method relative to support vector machine [15]. In subspace method, there are two notable points relative to support vector machine. One is fast processing in huge multi-class classification. The other is a possibility of a statistical quantitative assessment based on influence function. Therefore, in this paper, we apply a diagnostics based on influence function to a basic linear subspace method, CLAFIC method.

In statistics, influence function has been used to assess the influence of training sample for many target statistics in a lot of multivariate methods, and a statistical sensitivity analysis and diagnostics based on its function

have been developed by many researchers. Particularly, the diagnostics of discrimination based on the influence function was discussed by Campbell [1], Fung [4], and Huang et al. [9]. Using an influence function of the important statistics for a classifier, we can detect an influential observation for analysis results. If influential observations are outliers such as mislabeled data, we can largely improve the performance of a classifier by deleting them from the training data. In the field of pattern recognition, as I mentioned before, many extensions for the linear subspace method have been proposed, but the statistical evaluation of their classifiers on the basis of influence functions has not been established yet. In this paper, we focus on the Class-Featuring Information Compression (CLAFIC) method and propose a novel discriminant score for CLAFIC and its average. In addition, we define an important 
statistics and derive the empirical and sample influence functions. Through a simulation study and a real data analysis, we show that the diagnostics based on the influence function is also useful for the evaluation of a classifier in the linear subspace method.

\section{CLAFIC}

We denote the number of feature quantities or variables in a pattern vector $\boldsymbol{x}_{i}=\left(x_{i 1}, \ldots, x_{i p}\right)^{\mathrm{T}}$ as $p$, and we suppose that there are $K$ classes. In addition, we represent a training sample in the $k$-th class as $\boldsymbol{x}_{i}^{k}$, where $i$ ranges from 1 to $n_{k}$. $n_{k}$ is the number of samples in the $k$-th class. Then, the autocorrelation matrix of the training data in the $k$-th class is defined as follows:

$$
\hat{G}_{k}=\frac{1}{n_{k}} \sum_{i=1}^{n_{k}} \boldsymbol{x}_{i}^{k} \boldsymbol{x}_{i}^{k \mathrm{~T}} \quad(k=1, \ldots, K) .
$$

In the first step, we solve the eigenvalue problem for $\hat{G}_{k}$. Here, we denote the $p$ eigenvalues as $\hat{\lambda}_{1}^{k} \geq \cdots \geq \hat{\lambda}_{p}^{k} \geq 0$ and represent the normalized eigenvector corresponding to $\hat{\lambda}_{s}^{k}$ as $\hat{\boldsymbol{u}}_{s}^{k}(s=1, \ldots, p)$. Next, we calculate a projection matrix in the $k$-th class as follows:

$$
\hat{P}_{k}=\sum_{s=1}^{p_{k}} \hat{\boldsymbol{u}}_{s}^{k} \hat{\boldsymbol{u}}_{s}^{k \mathrm{~T}} \quad\left(1 \leq p_{k} \leq p\right),
$$

where $p_{k}$ is determined for the minimum value $m$ satisfying $\tau \leq \frac{\sum_{s=1}^{m} \hat{\lambda}_{s}^{k}}{\sum_{s=1}^{p} \hat{\lambda}_{s}^{k}}(1 \leq$ $m \leq p$ ) for a given $\tau$. In this regard, we suppose that the neighboring eigenvalues of $\hat{\lambda}_{p_{k}}^{k}$ are not equal. $\tau$ corresponds to a cumulative proportion in principal component analysis. Using a cross-validation method, we can determine the optimal value of $\tau$. Next, we project a test observation $\boldsymbol{x}^{*}$ into all subspaces based on (2) and calculate the squares of the projected norms,

$$
\boldsymbol{x}^{* \mathrm{~T}} \hat{P}_{k} \boldsymbol{x}^{*} \quad(k=1, \ldots, K) .
$$

We finally classify $\boldsymbol{x}^{*}$ into the class that gives the maximum value on (3). In this study, as a matter of convenience, we normalize all training and test data to one. 


\section{Influence functions}

In general data analysis, we necessarily face to an outlier problem that there is gross error in datasets to be analyzed. Therefore, as described in [8], to describe the structure best fitting the bulk of the data and to identify deviating data points or deviating substructures for further treatment are so important for data analysts. Their two things are the main aims of robust statistics [8]. In particularly, by using the influence function that was introduced by [7], we can detect outliers for a target statistics. In the field of statistics, a diagnostics based on the influence function has been developed as sensitivity analysis for many statistical multivariate methods [19]. On the other hand, sensitivity analysis based on the influence function for prediction accuracy on cross-validation in discriminant analysis has been less discussed. In general, in discrimination, if the average of the discriminant scores in each class is large, a magnitude of separation between classes is also large. Therefore, in this situation, we can expect that the prediction accuracy in discrimination is improved. The sign of the influence function for its statistics is also important for improving prediction accuracy. However, it seems that an application for the influence function and its sign has not been actively performed for statistical methods and pattern recognition methods. Then, in this section, we focus on a subspace method, CLAFIC method. We calculate the discriminant score for CLAFIC method and its average. We derive the influence function for the average.

In this section, we introduce three functions: the theoretical, empirical, and sample influence functions.

\subsection{Theoretical Influence Function}

Suppose $\theta_{k}\left(F^{1}, \ldots, F^{K}\right)(k=1, \ldots, K)$ are differentiable functionals of $F^{g}$, where $F^{g}(g=1, \ldots, K)$ are the cumulative distribution functions (cdf).

Then, the $g$-th perturbed cdf at $\boldsymbol{x}^{g}$ is defined as follows:

$$
\tilde{F}^{g}=(1-\varepsilon) F^{g}+\varepsilon \delta_{\boldsymbol{x}^{g}},
$$

where $\delta_{\boldsymbol{x}^{g}}$ is the cdf of a unit point mass at $\boldsymbol{x}^{g}$.

The theoretical influence function $(T I F)$ of $\theta_{k}$ at $\boldsymbol{x}^{g}$ is given by

$$
\operatorname{TIF}\left(\boldsymbol{x}^{g} ; \theta_{k}\right)=\lim _{\varepsilon \rightarrow 0} \frac{\theta_{k}\left(F^{1}, \ldots, \tilde{F}^{g}, \ldots, F^{K}\right)-\theta_{k}\left(F^{1}, \ldots, F^{g}, \ldots, F^{K}\right)}{\varepsilon} .
$$


In the above definition, we assume that the true distribution functions $F^{g}$ in all classes are known.

\subsection{Empirical Influence Function}

The empirical influence function $(E I F)$ is obtained by replacing $F^{g}$ in $T I F$ by $\hat{F}^{g}$, an empirical cdf [2]. With $E I F$, we can also evaluate the influence of each observation in the $g$-th class on an analysis result. We define

$$
\hat{F}^{g}=\frac{1}{n_{g}} \sum_{j=1}^{n_{g}} \delta_{x_{j}^{g}} \quad(g=1, \ldots, K) .
$$

Then, $E I F$ at a point of $\boldsymbol{x}_{r}^{g}\left(g=1, \ldots, K ; r=1, \ldots, n_{g}\right)$ is given by

$$
\operatorname{EIF}\left(\boldsymbol{x}_{r}^{g} ; \hat{\theta}_{k}\right)=\lim _{\varepsilon \rightarrow 0} \frac{\hat{\theta}_{k}\left(\hat{F}^{1}, \ldots, \tilde{\hat{F}}^{g}, \ldots, \hat{F}^{K}\right)-\hat{\theta}_{k}\left(\hat{F}^{1}, \ldots, \hat{F}^{g}, \ldots, \hat{F}^{K}\right)}{\varepsilon},
$$

where $\hat{\theta}_{k}$ is the empirical version of $\theta_{k}$ and $\tilde{\hat{F}}^{g}=(1-\varepsilon) \hat{F}^{g}+\varepsilon \delta_{\boldsymbol{x}_{r}^{g}}$.

\subsection{Sample Influence Function}

To evaluate the influence of observation for $\hat{\theta}_{k}$, we can use the sample influence function $(S I F)$ instead of $(7)$. SIF is calculated as follows:

$$
\operatorname{SIF}\left(\boldsymbol{x}_{r}^{g} ; \hat{\theta}_{k}\right)=-\left(n_{g}-1\right)\left\{\hat{\theta}_{k}\left(\hat{F}^{1}, \ldots, \hat{\hat{F}}^{g(-r)}, \ldots, \hat{F}^{K}\right)-\hat{\theta}_{k}\left(\hat{F}^{1}, \ldots, \hat{F}^{g}, \ldots, \hat{F}^{K}\right)\right\},
$$

where $\tilde{\hat{F}}^{g(-r)}=\left(1+\frac{1}{n_{g}-1}\right) \hat{F}^{g}-\frac{1}{n_{g}-1} \delta_{\boldsymbol{x}_{r}^{g}}$. The perturbation term $-\frac{1}{n_{g}-1}$ in the sample influence function corresponds to $\varepsilon$ in the empirical influence function. 


\section{Influence functions for CLAFIC}

In this section, we develop a discriminant score and its average for CLAFIC. Subsequently, we derive the influence functions for an essential part of the discriminant score.

\subsection{Discriminant score}

We define a discriminant score for $\boldsymbol{x}_{i}^{k}$ in the linear subspace method as follows:

$$
\hat{z}_{i}^{k}=\boldsymbol{x}_{i}^{k^{\mathrm{T}}} \hat{Q}_{k} \boldsymbol{x}_{i}^{k} \quad\left(1 \leq i \leq n_{k}\right),
$$

where

$$
\hat{Q}_{k}=\frac{1}{K-1}\left(K \hat{P}_{k}-\sum_{l=1}^{K} \hat{P}_{l}\right)=\frac{K}{K-1}\left(\hat{P}_{k}-\frac{1}{K} \sum_{l=1}^{K} \hat{P}_{l}\right) .
$$

In the above equations, $\hat{z}_{i}^{k}$ represents the magnitude of separation that $\boldsymbol{x}_{i}^{k}$ belonging to the $k$-th class relative to the other classes.

The average of the discriminant scores in each class is rewritten as

$$
\hat{Z}_{k}=\frac{1}{n_{k}} \sum_{i=1}^{n_{k}} \hat{z}_{i}^{k}
$$

If the value of $\hat{Z}_{k}$ is large enough, we can consider that the $k$-th class is well separated from the others. On the other hand, when there are many classes in a discriminant problem, we can also develop an indicator of the influence such that a perturbed observation gives a total sum of $\hat{Z}_{k} \mathrm{~s}$.

\subsection{EIF and SIF}

When we regard $\hat{Q}_{k}$ as $\hat{\theta}_{k}, E I F\left(\boldsymbol{x}_{r}^{g} ; \hat{Q}_{k}\right)$ is given by $\lim _{\varepsilon \rightarrow 0}\left[\hat{Q}_{k}\left(\hat{F}^{1}, \ldots, \tilde{\hat{F}}^{g}, \ldots, \hat{F}^{K}\right)-\right.$ $\left.\hat{Q}_{k}\left(\hat{F}^{1}, \ldots, \hat{F}^{g}, \ldots, \hat{F}^{K}\right)\right] / \varepsilon$, using the concept of perturbation analysis $[18$, 19]. 
If we perturb $\hat{Q}_{k}$ at a point of the $r$-th observation within the $g$-th class,

$$
\begin{aligned}
& \hat{Q}_{k}^{g r} \equiv \lim _{\varepsilon \rightarrow 0} \frac{\hat{Q}_{k}\left(\hat{F}^{1}, \ldots, \tilde{F}^{g}, \ldots, \hat{F}^{K}\right)-\hat{Q}_{k}\left(\hat{F}^{1}, \ldots, \hat{F}^{g}, \ldots, \hat{F}^{K}\right)}{\varepsilon} \\
& =\gamma \sum_{s=1}^{p_{g}} \sum_{t=p_{g}+1}^{p}\left(\hat{\lambda}_{s}^{g}-\hat{\lambda}_{t}^{g}\right)^{-1} \hat{\boldsymbol{u}}_{s}^{g \mathrm{~T}} \hat{G}_{g}^{g r} \hat{\boldsymbol{u}}_{t}^{g}\left(\hat{\boldsymbol{u}}_{s}^{g} \hat{\boldsymbol{u}}_{t}^{g \mathrm{~T}}+\hat{\boldsymbol{u}}_{t}^{g} \hat{\boldsymbol{u}}_{s}^{g \mathrm{~T}}\right)=\gamma P_{g}^{(1)},
\end{aligned}
$$

where $\hat{G}_{k}^{g r}$ is $\hat{\boldsymbol{x}}_{r}^{g} \hat{\boldsymbol{x}}_{r}^{g \mathrm{~T}}-\hat{G}_{g}\left(r=1, \ldots, n_{g}\right)$, and $p_{g}$ is the number of basis vectors for the projection matrix in the $g$-th class. When $g$ is equal to $k, \gamma$ is 1 . In other cases, $\gamma$ is equivalent to $-1 / K-1$. In (12), we denote the 1 st order differential at a point of $\boldsymbol{x}_{r}^{g}$ as the superscript (1).

\section{(Derivation)}

Let the rank of $\hat{G}_{g}$ be $p$ and its eigenvalues be distinct. In general, the matrix of $\hat{G}_{g}$ satisfies the following equation with the $j$-th eigenvalue and the associated eigenvector.

$$
\hat{G}_{g} \hat{\boldsymbol{u}}_{j}^{g}=\hat{\lambda}_{j}^{g} \hat{\boldsymbol{u}}_{j}^{g}, \quad j=1, \ldots, p .
$$

Hence, we get the following equation,

$$
\hat{G}_{g}^{(1)} \hat{\boldsymbol{u}}_{j}^{g}+\hat{G}_{g} \hat{\boldsymbol{u}}_{j}^{g(1)}=\hat{\lambda}_{j}^{g(1)} \hat{\boldsymbol{u}}_{j}^{g}+\hat{\lambda}_{j}^{g} \hat{\boldsymbol{u}}_{j}^{g(1)} .
$$

In the following discussion, we denote $\hat{G}_{g}^{g r}$ as $\hat{G}_{g}^{(1)}$. By multiplying $\hat{\boldsymbol{u}}_{j}^{g \mathrm{~T}}$ on the right side, we get

$$
\hat{G}_{g}^{(1)} \hat{\boldsymbol{u}}_{j}^{g} \hat{\boldsymbol{u}}_{j}^{g \mathrm{~T}}=\hat{\lambda}_{j}^{g(1)} \hat{\boldsymbol{u}}_{j}^{g} \hat{\boldsymbol{u}}_{j}^{g \mathrm{~T}} .
$$

Then, we obtain the following relationship,

$$
\sum_{j=1}^{p} \hat{G}_{g}^{(1)} \hat{\boldsymbol{u}}_{j}^{g} \hat{\boldsymbol{u}}_{j}^{g \mathrm{~T}}=\sum_{j=1}^{p} \hat{\lambda}_{j}^{(1)} \hat{\boldsymbol{u}}_{j}^{g} \hat{\boldsymbol{u}}_{j}^{g \mathrm{~T}} .
$$

Therefore,

$$
\hat{G}_{g}^{(1)}=\sum_{j=1}^{p} \hat{\lambda}_{j}^{g(1)} \hat{\boldsymbol{u}}_{j}^{g} \hat{\boldsymbol{u}}_{j}^{g \mathrm{~T}} .
$$


From (17),

$$
\hat{\boldsymbol{u}}_{s}^{g \mathrm{~T}} \hat{G}_{g}^{(1)} \hat{\boldsymbol{u}}_{s}^{g}=\hat{\lambda}_{s}^{g^{(1)}},
$$

where we focus only on $s=1, \ldots, p_{g}$.

Based on [17], the empirical influence function of the normalized eigenvector $\hat{\boldsymbol{u}}_{s}^{g}$ that associates with the eigenvalue $\hat{\lambda}_{s}^{g}$ is given by

$$
\hat{\boldsymbol{u}}_{s}^{g(1)}=\left(\hat{G}_{g}+\hat{\boldsymbol{u}}_{s}^{g} \hat{\boldsymbol{u}}_{s}^{g \mathrm{~T}}-\hat{\lambda}_{s}^{g} \mathbf{I}_{p}\right)^{-1}\left(\hat{\lambda}_{s}^{g(1)} \mathbf{I}_{p}-\hat{G}_{g}^{(1)}\right) \hat{\boldsymbol{u}}_{s}^{g},
$$

where $\mathbf{I}_{p}$ is the identity matrix of order $p$.

In (19), we substitute $\sum_{j=1}^{p} \hat{\lambda}_{j}^{g} \hat{\boldsymbol{u}}_{j}^{g} \hat{\boldsymbol{u}}_{j}^{g \mathrm{~T}}$ and $\sum_{j=1}^{p} \hat{\boldsymbol{u}}_{j}^{g} \hat{\boldsymbol{u}}_{j}^{g \mathrm{~T}}$ into $\hat{G}_{g}$ and $\mathbf{I}_{p}$, respectively.

Then,

$$
\hat{\boldsymbol{u}}_{s}^{g(1)}=\left[\sum_{j=1}^{p}\left(\hat{\lambda}_{j}^{g}-\hat{\lambda}_{s}^{g}\right) \hat{\boldsymbol{u}}_{j}^{g} \hat{\boldsymbol{u}}_{j}^{g \mathrm{~T}}+\hat{\boldsymbol{u}}_{s}^{g} \hat{\boldsymbol{u}}_{s}^{g \mathrm{~T}}\right]^{-1}\left[\hat{\lambda}_{s}^{g(1)} \hat{\boldsymbol{u}}_{s}^{g}-\hat{G}_{g}^{(1)} \hat{\boldsymbol{u}}_{s}^{g}\right] .
$$

We apply the Sherman-Morrison formula and the following the relationship to (20).

$$
\left[\sum_{j=1}^{p}\left(\hat{\lambda}_{j}^{g}-\hat{\lambda}_{s}^{g}\right) \hat{\boldsymbol{u}}_{j}^{g} \hat{\boldsymbol{u}}_{j}^{g \mathrm{~T}}\right]^{-1}\left(\hat{\boldsymbol{u}}_{s}^{g} \hat{\boldsymbol{u}}_{s}^{g \mathrm{~T}}\right)^{-1}=\left[\sum_{j=1}^{p}\left(\hat{\lambda}_{j}^{g}-\hat{\lambda}_{s}^{g}\right) \hat{\boldsymbol{u}}_{j}^{g} \hat{\boldsymbol{u}}_{j}^{g \mathrm{~T}} \hat{\boldsymbol{u}}_{s}^{g} \hat{\boldsymbol{u}}_{s}^{g \mathrm{~T}}\right]^{-1}=0 .
$$

Then,

$$
\hat{\boldsymbol{u}}_{s}^{g(1)}=\sum_{j=1, j \neq s}^{p}\left(\hat{\lambda}_{s}^{g}-\hat{\lambda}_{j}^{g}\right)^{-1} \hat{\boldsymbol{u}}_{j}^{g} \hat{\boldsymbol{u}}_{j}^{g \mathrm{~T}} \hat{G}_{g}^{(1)} \hat{\boldsymbol{u}}_{s}^{g}
$$

In CLAFIC, we consider the perturbation for $\hat{P}_{g}$ at a point of $\boldsymbol{x}_{r}^{g}$. $\hat{P}_{g}$ is calculated by

$$
\hat{P}_{g}^{(1)}=\sum_{s=1}^{p_{g}} \hat{\boldsymbol{u}}_{s}^{g(1)} \hat{\boldsymbol{u}}_{s}^{g \mathrm{~T}}+\hat{\boldsymbol{u}}_{s}^{g} \hat{\boldsymbol{u}}_{s}^{g(1)^{\mathrm{T}}} .
$$

We substitute (22) into (23) so that

$$
\begin{aligned}
\hat{P}_{g}^{(1)}= & \sum_{s=1}^{p_{g}} \sum_{j=1, j \neq s}^{p}\left(\hat{\lambda}_{s}^{g}-\hat{\lambda}_{j}^{g}\right)^{-1} \hat{\boldsymbol{u}}_{j}^{g} \hat{\boldsymbol{u}}_{j}^{g \mathrm{~T}} \hat{G}_{g}^{(1)} \hat{\boldsymbol{u}}_{s}^{g} \hat{\boldsymbol{u}}_{s}^{g \mathrm{~T}} \\
& +\hat{\boldsymbol{u}}_{s}^{g} \hat{\boldsymbol{u}}_{s}^{g \mathrm{~T}}\left(\hat{\lambda}_{s}^{g}-\hat{\lambda}_{j}^{g}\right)^{-1} \hat{G}_{g}^{(1)} \hat{\boldsymbol{u}}_{j}^{g} \hat{\boldsymbol{u}}_{j}^{g \mathrm{~T}}
\end{aligned}
$$




$$
=\sum_{s=1}^{p_{g}} \sum_{j=p_{g}+1}^{p}\left(\hat{\lambda}_{s}^{g}-\hat{\lambda}_{j}^{g}\right)^{-1} \hat{\boldsymbol{u}}_{s}^{g \mathrm{~T}} \hat{G}_{g}^{(1)} \hat{\boldsymbol{u}}_{j}^{g}\left(\hat{\boldsymbol{u}}_{s}^{g} \hat{\boldsymbol{u}}_{j}^{g \mathrm{~T}}+\hat{\boldsymbol{u}}_{j}^{g} \hat{\boldsymbol{u}}_{s}^{g \mathrm{~T}}\right)
$$

$\operatorname{SIF}\left(\boldsymbol{x}_{r}^{g} ; \hat{Q}_{k}\right)(g=1, \ldots, K)$ is $-\left(n_{g}-1\right)\left(\hat{Q}_{k}^{g(-r)}-\hat{Q}_{k}\right) . \hat{Q}_{k}^{g(-r)} \operatorname{denotes} \hat{Q}_{k}$ without the $r$-th observation in the $g$-th class. We write $-\left(n_{g}-1\right)\left(\hat{Q}_{k}^{g(-r)}-\right.$ $\left.\hat{Q}_{k}\right)$ as $\hat{Q}_{k g(-r)}$. By considering the loadings of high order terms in $\varepsilon$, we can enhance the power of approximation for $\hat{Q}_{k}^{g r}$ and $\hat{Q}_{k g(-r)}$.

\subsection{Single-case diagnostics}

In sensitivity analysis in statistics, single-case and multiple-case diagnostics were proposed for mainly exploratory multivariate methods [19]. The former is based on a single observation and the latter on multiple observations. In this paper, we focus on single-case diagnostics for a discriminant method in pattern recognition. Since $E I F\left(\boldsymbol{x}_{r}^{g} ; \hat{Q}_{k}\right)$ and $S I F\left(\boldsymbol{x}_{r}^{g} ; \hat{Q}_{k}\right)$ are vectors, we summarize them into the scalar measures to clarify the influence of the discriminant boundaries.

By the effect of perturbing $\hat{Q}_{k}$ at a point $\boldsymbol{x}_{i}^{g}, \hat{Z}_{k}$ equal to the average of the discriminant scores in each class is also affected as follows.

In the case of $\hat{Q}_{k}^{g r}$,

$$
\begin{aligned}
& \hat{Z}_{k}=\hat{Z}_{k}+\varepsilon \hat{Z}_{k}^{g r}+O\left(\varepsilon^{2}\right), \\
& \hat{Z}_{k}^{g r}=\frac{1}{n_{k}} \sum_{i=1}^{n_{k}} x_{i}^{k^{\mathrm{T}}} \hat{Q}_{k}^{g r} \boldsymbol{x}_{i}^{k} .
\end{aligned}
$$

In the case of $\hat{Q}_{k g(-r)}$,

$$
\hat{Z}_{k}^{g(-r)}=\frac{1}{n_{k}} \sum_{i=1}^{n_{k}} \boldsymbol{x}_{i}^{k^{\mathrm{T}}} \hat{Q}_{k g(-r)} \boldsymbol{x}_{i}^{k} .
$$

Here, we can consider $\hat{Z}_{k}^{g r}$ and $\hat{Z}_{k}^{g(-r)}$ as the effects for a degree of separation between $k$-class and other classes. We plot $\hat{Z}_{k}^{g r}$ or $\hat{Z}_{k}^{g(-r)}$ for each class along the index $r$ in a horizontal $x$-axis, and we can detect a large influential observation. Here we pay attention to the following two things. One is a magnitude of the value of the influence function. The other is the sign of the influence function. The former shows the power of a target training sample 
for prediction accuracy. The latter represents whether the prediction accuracy is improved or not by perturbing the training sample. In this paper, the negative value of the influence function for the average of the discriminant scores represents the influence of the improvement of prediction by perturbation.

In EIF, we evaluate the influence of training sample for a target statistics by the first derivative at training sample. On the other hand, in $S I F$, we assess the influence of training sample for a target statistics with the deleting effect corresponding to the first derivative effect. A large influential observation has a different some properties relative to other training samples. Therefore, at the influential sample, the approximation between SIF and $E I F$ does not often work well, but the discrepancy gives us a finding that there is a large influential training sample.

In general, when we perform a diagnostics, we fix the tuning parameter optimized before diagnostics. Then, with the same tuning parameter before diagnostics, we perturb the empirical cumulative distribution function at the training sample and calculate the empirical or sample influence function. If we achieve the improvement of the classifier in the discrimination, after deleting the outliers or reducing the weight of the outliers, we have to recalculate the optimal value of $\tau$ again.

\section{Simulation studies}

When we perform a discrimination on pixel image data, we always encounter high-dimensional datasets. For a high-dimensional analysis, preprocessing is performed before discrimination in pattern recognition. For example, self-featuring information compression is a representative processing and feature extraction method with which we can reduce a high dimension to a relatively small dimension on the basis of effective dimensions for discrimination. In this study, we set two discrimination problems and show their simulation results: a three-class discrimination problem (Case 1) and a four-class discrimination problem (Case 2). In Case 1 and Case 2, we assume that there are three mislabeled training samples that are crucial influential samples in training data. The numbers of feature dimensions in Case 1 and Case 2 are 200 and 1,000, respectively. In the following subsections, for each simulation study, we give a detailed explanation of the setting status and analysis results for Case 1 and Case 2 . 


\subsection{Study of Case 1}

The data in Class 1 and Class 2 followed a mixture of two multivariate normal distributions whereas the data in Class 3 followed a mixture of three multivariate normal distributions. We first set the population mean vectors in each class by choosing 200 integers from 0 to 255 by simple random sampling. Then, for each mean vector, 200 sets of noise data were generated using a multivariate normal distribution and then added to each element of the mean vector 10 times and used the result to calculate the covariance matrix of the population. In this study, a mean vector of the population in Class 1 was similar to that of the population in Class 3 , and the two mean vectors of population in Class 2 were also similar to one of the mean vectors of the population in Class 1. In addition, a mean vector of the population in Class 3 was set to be slightly similar to a mean vector of the population in Class 1. The distributions of the noise data for the mean vectors in each class are shown in Table 1.

Table 1: Distributions for the mean vectors in each class

\begin{tabular}{|c|c|}
\hline Class & Distribution \\
\hline Class 1 & $N(0,40), \quad N(0,30)$ \\
Class 2 & $N(0,20), \quad N(0,35)$ \\
Class 3 & $N(0,20), \quad N(0,35), \quad N(0,20)$ \\
\hline
\end{tabular}

We generated 30 datasets for each component of each class. For each class, we used these datasets to select 30 training samples on the basis of simple random sampling. In this study, as previously mentioned, we regarded mislabeled data as outliers. We replaced the first and second training samples in Class 1 with the training samples generated from one of the components of Class 2 and Class 3, respectively. In addition, we substituted the 29th training sample in Class 2 with the training sample generated from one of the components of Class 1. We first developed the classifier in CLAFIC using the optimal tuning parameter $\tau$ obtained by leave-one-out cross-validation. To obtain the optimal value of $\tau$, we shifted the value from 0.900 to 0.999 in increments of 0.001 (see Figure 1). The optimal value of $\tau$ was determined to be 0.915 . The numbers of basis vectors in Class 1, Class 2, and Class 3 were 1,1 , and 2 , respectively. With this threshold, we developed the optimal 


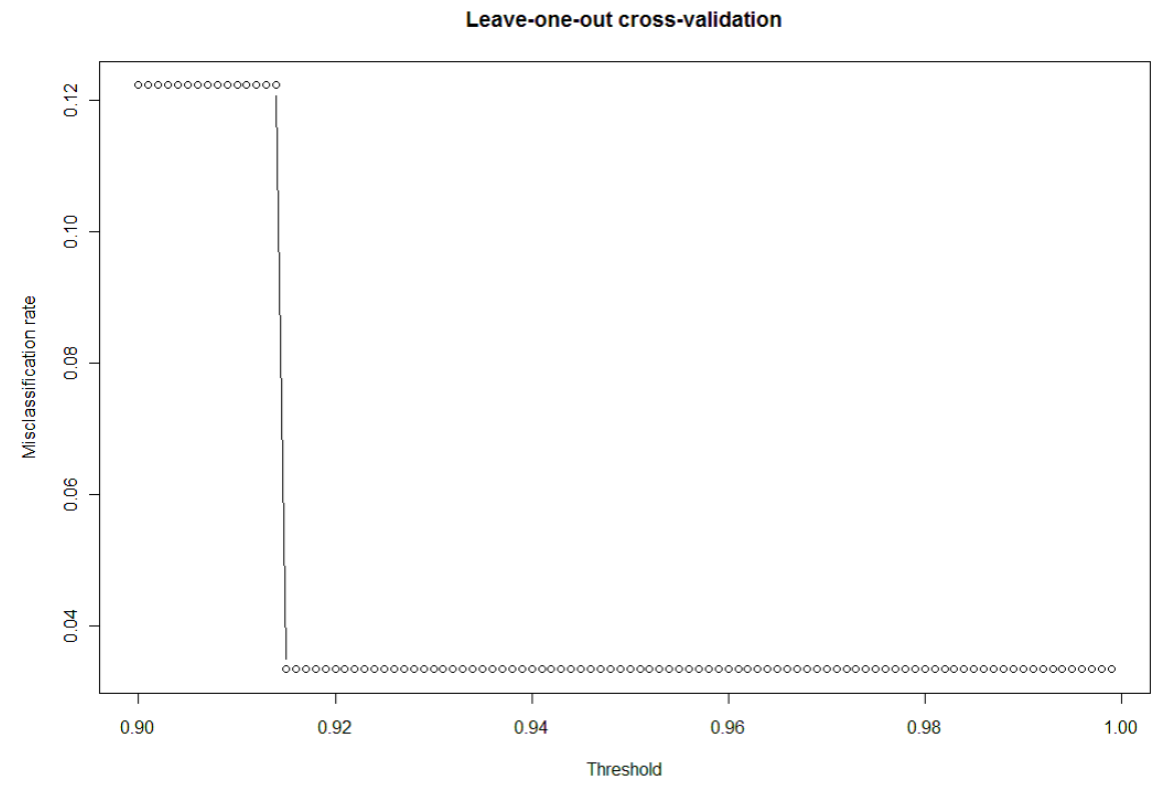

Figure 1: Model selection in CLAFIC

classifier in CLAFIC method and applied the classifier to the 30 training and 3,000 test datasets in each class. The results are shown in Tables 2 and 3. Next, before applying our single-case diagnostics to this situation,

Table 2: Results of training data before diagnostics $(\tau=0.915)$

\begin{tabular}{cccc}
\hline Class & Class 1 & Class 2 & Class 3 \\
\hline Class 1 & 28 & 1 & 1 \\
Class 2 & 1 & 29 & 0 \\
Class 3 & 0 & 0 & 30 \\
\hline
\end{tabular}

we summarized the results using leave-one-out cross-validation (see Table 4). Table 4 shows that the optimal classifier precisely rejected three sets of mislabeled data. However, from the results of the test data, we can also confirm that the classifier is slightly affected by some artificial mislabeled data. To investigate the details, we applied our approach to the optimal 
Table 3: Results of test data before diagnostics $(\tau=0.915)$

\begin{tabular}{cccc}
\hline Class & Class 1 & Class 2 & Class 3 \\
\hline Class 1 & 2995 & 5 & 0 \\
Class 2 & 0 & 3000 & 0 \\
Class 3 & 0 & 0 & 3000 \\
\hline
\end{tabular}

Table 4: Results of leave-one-out cross-validation before diagnostics $(\tau=0.915)$

\begin{tabular}{cccc}
\hline Class & Class 1 & Class 2 & Class 3 \\
\hline Class 1 & 28 & 1 & 1 \\
Class 2 & 1 & 29 & 0 \\
Class 3 & 0 & 0 & 30 \\
\hline
\end{tabular}

classifier. In Figures 2-4, we show the sample influence function of the average of the discriminant scores relative to the empirical influence function of the discriminant score average in each class.

In the empirical influence function, $\operatorname{EIF}\left(\boldsymbol{x}_{r}^{g} ; \hat{Z}_{k}\right)(k=g)$ is equal to 0 , and therefore, we plotted the sample influence function relative to the empirical influence function in Class $k(\neq g)$. From Figures 2-4, we can see that in this situation, the approximation of the sample and empirical influence functions is good in all classes. However, in the second training sample in Figure 2, there is also a discrepancy between $S I F$ and $E I F$. This may imply that the training sample at this point of discrepancy corresponds to a large influential training sample. If we consider the approximation of $S I F$ and EIF with both the fist and second order terms, we can enhance their approximation. In Figures 5-7, we plotted the sample influence functions after perturbing the training samples in each class.

From Figure 5, we can see that the deletion of the first and second training samples in Class 1 significantly affects the discrimination. In our diagnostics, the large negative value of $S I F$ or $E I F$ for a training sample indicates improvement in prediction accuracy by deleting the target sample. On the other hand, the large positive value of SIF or EIF for a training sample shows reduction in prediction accuracy by deleting the target sample. There- 
fore, if we exclude the first and second training samples in Class 1, we can expect improvement in the prediction accuracy of Class 2 and Class 3, respectively. Moreover, from Figure 6, we can consider the 29th training sample as a suspected datum of a mislabeled sample. As seen above, we detected three large influential training samples. The number of all possible combinations of subsets for deleting these influential samples is seven $\left(2^{3}-1\right)$. Therefore, by deleting each subset at the optimal threshold (0.915), we calculated the misclassification rates on the basis of leave-one-out cross-validation. The results are shown in Table 5. On the basis of Table 5, the optimal threshold

Table 5: Results of misclassification rate by deleting training subset $(\tau=0.915)$

\begin{tabular}{cc}
\hline Deleting training subset & Misclassification rate \\
\hline$\left\{\boldsymbol{x}_{1}^{1}\right\}$ & $2.247 \%$ \\
$\left\{\boldsymbol{x}_{2}^{1}\right\}$ & $2.247 \%$ \\
$\left\{\boldsymbol{x}_{29}^{2}\right\}$ & $2.247 \%$ \\
$\left\{\boldsymbol{x}_{1}^{1}, \boldsymbol{x}_{2}^{1}\right\}$ & $1.136 \%$ \\
$\left\{\boldsymbol{x}_{1}^{1}, \boldsymbol{x}_{29}^{2}\right\}$ & $1.136 \%$ \\
$\left\{\boldsymbol{x}_{2}^{1}, \boldsymbol{x}_{29}^{2}\right\}$ & $1.136 \%$ \\
$\left\{\boldsymbol{x}_{1}^{1}, \boldsymbol{x}_{2}^{1}, \boldsymbol{x}_{29}^{2}\right\}$ & $0.000 \%$ \\
\hline
\end{tabular}

was recalculated by deleting $\left\{\boldsymbol{x}_{1}^{1}, \boldsymbol{x}_{2}^{1}, \boldsymbol{x}_{29}^{2}\right\}$. The value of the recalculated optimal threshold was also 0.915 . When we deleted $\left\{\boldsymbol{x}_{1}^{1}, \boldsymbol{x}_{2}^{1}, \boldsymbol{x}_{29}^{2}\right\}$ and developed the classifier on the basis of the recalculated optimal threshold, the numbers of basis vectors in Class 1, Class 2, and Class 3 were also 1, 1, and 2 , respectively. The results of the test data are shown in Table 6 .

Table 6: Results of test data after diagnostics $(\tau=0.915)$

\begin{tabular}{cccc}
\hline Class & Class 1 & Class 2 & Class 3 \\
\hline Class 1 & 3000 & 0 & 0 \\
Class 2 & 0 & 3000 & 0 \\
Class 3 & 0 & 0 & 3000 \\
\hline
\end{tabular}




\subsection{Study of Case 2}

In Case 2, the data in Class 1 and Class 2 follow a mixture of two multivariate normal distributions, whereas the data in Class 3 and Class 4 follow a mixture of three multivariate normal distributions. For each component of each class, we set the mean vector and covariance matrix using the same procedure followed for Case 1 . We set one mean vector of the population in Class 2 to be similar to one of the mean vectors of the population in Class 1. In addition, we also set the other mean vector of the population in Class 2 to be slightly similar to the other mean vector of the population in Class 1. One of the mean vectors of the population in Class 1 was set to be somewhat close to one of the mean vectors of the population in Class 3 . The two mean vectors of the population in Class 3 were set to be similar to one of the mean vectors of the population in Class 4 and to be close to the other mean vector of the population in Class 4. Moreover, the two mean vectors of the population in Class 4 were set to be close to one of the mean vectors of the population in Class 3 and the other mean vector of the population in Class 3. The noise data distributions for the mean vectors in each class are shown in Table 7. As in Case 1, we generated 15 training and 1,000

Table 7: Distributions for the mean vectors in each class

\begin{tabular}{|c|c|}
\hline Class & Distribution \\
\hline Class 1 & $N(0,50), \quad N(0,20)$ \\
Class 2 & $N(0,40), \quad N(0,30)$ \\
Class 3 & $N(0,20), \quad N(0,30), \quad N(0,10)$ \\
Class 4 & $N(0,20), \quad N(0,30), \quad N(0,35)$ \\
\hline
\end{tabular}

test datasets with equal probability in all components of each class. Then, we replaced the 7 th and 14th training samples in Class 1 with the training samples generated from components of Class 2 and Class 3, respectively. In addition, we also substituted the 15th training sample in Class 4 with the training sample generated from a component of Class 3. In this situation, to obtain the optimal threshold, we shifted the value of $\tau$ from 0.900 to 0.999 in increments of 0.001 (see Figure 8). On the basis of Figure 8, the value of $\tau$ was determined to be 0.977 . When we developed the classifier in CLAFIC using the optimal threshold, the numbers of basis vectors in Class 1, Class 
2, Class 3, and Class 4 were 2, 1, 2, and 2, respectively. We applied the optimized classifier to the 15 training and 3,000 test datasets in each class. Then, we obtained the results of the training and test datasets, as shown in Table 8 and Table 9, respectively. Moreover, we applied leave-one-out

Table 8: Results of training data before diagnostics ( $\tau=0.977$ )

\begin{tabular}{ccccc}
\hline Class & Class 1 & Class 2 & Class 3 & Class 4 \\
\hline Class 1 & 14 & 1 & 0 & 0 \\
Class 2 & 0 & 15 & 0 & 0 \\
Class 3 & 0 & 0 & 15 & 0 \\
Class 4 & 0 & 0 & 0 & 15 \\
\hline
\end{tabular}

Table 9: Results of test data before diagnostics ( $\tau=0.977$ )

\begin{tabular}{ccccc}
\hline Class & Class 1 & Class 2 & Class 3 & Class 4 \\
\hline Class 1 & 1000 & 0 & 0 & 0 \\
Class 2 & 2 & 998 & 0 & 0 \\
Class 3 & 10 & 0 & 935 & 55 \\
Class 4 & 0 & 0 & 0 & 1000 \\
\hline
\end{tabular}

cross-validation to the classifier. The results are shown in Table 10. The

Table 10: Results of leave-one-out cross-validation before diagnostics ( $\tau=0.977$ )

\begin{tabular}{ccccc}
\hline Class & Class 1 & Class 2 & Class 3 & Class 4 \\
\hline Class 1 & 13 & 1 & 1 & 0 \\
Class 2 & 0 & 15 & 0 & 0 \\
Class 3 & 0 & 0 & 15 & 0 \\
Class 4 & 0 & 0 & 0 & 15 \\
\hline
\end{tabular}

results are shown in Table 10. In Tables 8-10, we can see that the classifier 
was affected by some mislabeled data. Therefore, we applied our diagnostics to this situation. Figure 9-12 show that the sample influence function of the average of the discriminant scores relative to the empirical influence function of the discriminant score average in each class.

In this situation, for each class, we can confirm that the approximations of the sample and empirical influence functions are good. Here, we can also see that the 14th training sample in Class 1 and the 15 th training sample in Class 4 are large influential training samples. We plotted the sample influence functions by perturbing the training samples in each class in Figures 13 to 16. From Figure 13, we can improve the discrimination in Class 2 by deleting the 7 th training sample in Class 1 . In addition, we can improve the prediction accuracy in Class 3 and Class 4 by deleting the 14th training sample in Class 1. Moreover, we can improve the prediction accuracy in Class 1, Class 2, and Class 3 by deleting the 15th training sample. We could also detect three large influential samples in Case 2. Therefore, for all possible combination subsets involving the deletion of three large influential samples, we calculated the misclassification rates on the basis of leave-one-out crossvalidation at the optimal threshold $(0.977)$. The results are shown in Table 11. Based on Table 11, deleting $\left\{\boldsymbol{x}_{7}^{1}, \boldsymbol{x}_{14}^{1}\right\}$ or $\left\{\boldsymbol{x}_{7}^{1}, \boldsymbol{x}_{14}^{1}, \boldsymbol{x}_{15}^{4}\right\}$ realized the best prediction accuracy results. Finally, in each case, the optimal threshold was recalculated by shifting the value of $\tau$ from 0.900 to 0.999 in increments of 0.001 . When we deleted $\left\{\boldsymbol{x}_{7}^{1}, \boldsymbol{x}_{14}^{1}, \boldsymbol{x}_{15}^{4}\right\}$, the optimal value of $\tau$ was 0.900 and the misclassification rate based on leave-one-out cross-validation was zero. At this threshold, the numbers of basis vectors in Class 1, Class 2, Class 3, and Class 4 were 1, 1, 1, and 1, respectively. On the other hand, when we excluded $\left\{\boldsymbol{x}_{7}^{1}, \boldsymbol{x}_{14}^{1}\right\}$, the misclassification rate was $1.724 \%$ with a threshold of 0.900. Then, we determined that we can enhance the prediction accuracy with fewer basis vectors if we delete $\left\{\boldsymbol{x}_{7}^{1}, \boldsymbol{x}_{14}^{1}, \boldsymbol{x}_{15}^{4}\right\}$. Therefore, we determined $\left\{\boldsymbol{x}_{7}^{1}, \boldsymbol{x}_{14}^{1}, \boldsymbol{x}_{15}^{4}\right\}$ to be the mislabeled subset. The results of the test data based on the threshold of 0.900 are given in Table 12 .

Using the above procedure, we could precisely detect all mislabeled data and enhance the prediction accuracy of the classifier in CLAFIC method. 
Table 11: Results of misclassification rate by deleting training subset $(\tau=0.997)$

\begin{tabular}{cc}
\hline Deleting subset & Misclassification rate \\
\hline$\left\{\boldsymbol{x}_{7}^{1}\right\}$ & $1.695 \%$ \\
$\left\{\boldsymbol{x}_{14}^{1}\right\}$ & $1.695 \%$ \\
$\left\{\boldsymbol{x}_{15}^{4}\right\}$ & $3.390 \%$ \\
$\left\{\boldsymbol{x}_{7}^{1}, \boldsymbol{x}_{14}^{1}\right\}$ & $0.000 \%$ \\
$\left\{\boldsymbol{x}_{7}^{1}, \boldsymbol{x}_{15}^{4}\right\}$ & $1.724 \%$ \\
$\left\{\boldsymbol{x}_{14}^{1}, \boldsymbol{x}_{15}^{4}\right\}$ & $1.724 \%$ \\
$\left\{\boldsymbol{x}_{7}^{1}, \boldsymbol{x}_{14}^{1}, \boldsymbol{x}_{15}^{4}\right\}$ & $0.000 \%$ \\
\hline
\end{tabular}

Table 12: Results of test data after diagnostics $(\tau=0.900)$

\begin{tabular}{ccccc}
\hline Class & Class 1 & Class 2 & Class 3 & Class 4 \\
\hline Class 1 & 1000 & 0 & 0 & 0 \\
Class 2 & 0 & 1000 & 0 & 0 \\
Class 3 & 0 & 0 & 1000 & 0 \\
Class 4 & 0 & 0 & 0 & 1000 \\
\hline
\end{tabular}

\section{Real data analysis}

As a real data analysis, we used the MNIST database of handwritten digits (http://yann.lecun.com/exdb/mnist/). In this database, there is a dataset composed of training and test samples in terms of ten handwritten digits ("0", "1", "2", "3", "4", "5", "6", "7", "8", "9"). We used 8-bit grayscale images of "0" through "9" (http://www.cs.nyu.edu/ roweis/data.html).

All handwritten digits are size normalized, and centered in a fixed size image where the center of gravity of the intensity lies at the center of the image with $28 \times 28$ pixels [3]. The digits "1", "7", and "9" are so similar each other in some characteristics. Therefore, by considering the fact that the probability of the occurrence of mislabeling is high in the discrimination of "1", "7", and "9", we focused on a three-class discrimination for these handwritten digits. The number of the training samples in "1", "7", and "9" are 6742, 6265, and 5949 , respectively. To show the performance of the proposed sensitivity anal- 
ysis based on influence functions clearly, at 250 sample intervals, we selected 15 samples as training samples from the training dataset in each class. For the test data, we used all test data of Class "1", Class "7", and Class "9" shown in the MNIST database. We first calculated the optimal threshold $\tau$ based on leave-one-out cross-validation. We varied the value from 0.600 to 0.990 in intervals of 0.010 . Then, the optimal $\tau$ was 0.740 . The number of the optimal basis vectors in Class "1", Class "7", and Class "9" were two, three, and four, respectively. We next applied the developed classifier based on CLAFIC method with the optimal $\tau$ to the training and test data, respectively. From Table 13, we can see that the prediction accuracy in training data was partly affected by large influential training samples between Class "7" and Class "9". In this situation, we applied our proposed diagnostics to the discriminant model. Based on Figures 17-19, we considered the deletion of a few of essential and large influential training samples. In the analysis of our diagnostics, we can see that there were some training samples that are difficult to determine. For example, we can see that the 3rd training sample in Class "7", the 3rd training sample in Class " 9 , and the 13 th training sample in Class "9" have the tendency decreasing the prediction accuracy of the discrimination between Class "7" and Class "9". In this case, when we deleted $\left\{\boldsymbol{x}_{3}^{7}, \boldsymbol{x}_{3}^{9}, \boldsymbol{x}_{13}^{9}\right\}$, the misclassification rate based on leave-one-out crossvalidation was zero and the optimal value of $\tau$ was 0.700 . The number of the optimal basis vectors in Class "1", Class "7", and Class "9" were changed into two, three, and three, respectively. The results of test data after diagnostics are shown in Table 15. Total misclassification rate was changed from $8.008 \%$ to $6.967 \%$.

We also studied the performance of our diagnostics for a real data analysis in a specific situation, and confirmed the effectiveness of our diagnostics. In general, by using not only leave-one-out cross-validation but also $K$-fold cross-validation, we have to detect a small number of large influential training samples based on our proposed diagnostics. In this study, to compare the analysis result of this real data analysis with the analysis results of the simulation studies of Cases 1 and 2, we only used leave-one out-cross validation in the discrimination of handwritten digits. Based on the digit pictures shown in the site (http://www.cs.nyu.edu/ roweis/data.html), we also investigated the characteristics of these large influential digits. Then, we could found out that some characteristics of these influential handwritten digits were visually similar each other. 
Table 13: Results of training data before diagnostics $(\tau=0.740)$

\begin{tabular}{cccc}
\hline Class & "1" & "7" & "9" \\
\hline "1" & 15 & 0 & 0 \\
"7" & 0 & 14 & 1 \\
"9" & 0 & 0 & 15 \\
\hline
\end{tabular}

Table 14: Results of test data before diagnostics $(\tau=0.740)$

\begin{tabular}{cccc}
\hline Class & "1" & "7" & "9" \\
\hline "1" & 1128 & 4 & 3 \\
"7" & 35 & 855 & 138 \\
"9" & 14 & 60 & 935 \\
\hline
\end{tabular}

Table 15: Results of test data after diagnostics $(\tau=0.700)$

\begin{tabular}{cccc}
\hline Class & "1" & "7" & "9" \\
\hline "1" & 1129 & 4 & 2 \\
"7" & 37 & 933 & 58 \\
"9" & 17 & 103 & 889 \\
\hline
\end{tabular}

\section{Discussion and conclusion}

In this paper, we focused on CLAFIC method, which is a linear subspace method. To assess the influence of the training sample on analysis results and the prediction accuracy in CLAFIC method, we defined the discriminant score and its average and derived the sample and empirical influence functions. Using clear examples, we verified the performance of our approach. Using the modified classifier, we reduced the total number of misclassification rates in test data. Moreover, by adding our single-case diagnostics to the summarized table using leave-one-out cross-validation, we visually confirmed the influential direction of each training sample for the degree of separation between classes. In our diagnostics, we focused on the large negative value 
of the influence function of the discriminant score average in each class and detected the essential and influential training samples. For the large influential training samples that were detected, we calculated all their possible combination subsets. Therefore, we do not have to evaluate the influence of all possible combination subsets for all training samples.

In this study, to focus on one central theme, we only considered singlecase assessment in CLAFIC method. However, further study needs to be conducted to investigate additional linear subspace methods. In future, we plan to develop a diagnostics framework for extended linear subspace methods.

\section{Acknowledgments}

I would like to thank prof. Yutaka Tanaka and prof. Masahiro Mizuta for their helpful comments. This work was partly supported by the Core Research of Evolutional Science and Technology (CREST) in Japan Science and Technology Agency (Project: Alliance between Mathematics and Radiology).

\section{References}

[1] A.N. Campbell, The influence function as an aid in outlier detection in discriminant analysis, Applied Statistics 27 (1978) 251-258.

[2] F. Critchley, Influence in principal components analysis, Biometrika 72 (3) (1985) 627-636.

[3] L. Deng, The MNIST database of handwritten digit images for machine learning research, IEEE Signal Processing Magazine 29 (6) (2012) 141142 .

[4] W.K. Fung, Diagnosing influential observations in quadratic discriminant analysis, Biometrics 52 (1996) 1235-1241.

[5] S. Gunal, R. Edizkan, Subspace and feature selection for pattern recognition, Information Sciences 178 (19) (2008) 3716-3726.

[6] M.B. Gülmezoğlz, V. Dzhafarov, R. Edizkan, A. Barkana, The common vector approach and its comparison with other subspace methods in case of sufficient data, Computer Speech and Language 21 (2) (2007) 266-281. 
[7] F.R. Hampel, The influence curve and its role in robust estimation, Journal of the American Statistical Association 69 (1974) 383-393.

[8] F.R. Hampel, E.M. Ronchetti, P.J. Rousseeuw, W.A. Stahel, Robust statistics: The approach based on influence functions, John Wiley \& Sons, New York, 1986.

[9] Y. Huang, T.L. Kao, T.H. Wang, Influence functions and local influence in linear discriminant analysis, Computational Statistics and Data Analysis 51 (2007) 3844-3861.

[10] J. Karhunen, E. Oja, Some comments on the subspace methods of classification, in: Proceedings of the 5th International Conference on Pattern Recognition, 1980, pp. 1191-1194.

[11] T. Kitamura, S. Takeuchi, S. Abe, K. Fukui, Subspace-based support vector machines for pattern recognition, Neural Networks 22 (2009) 558567.

[12] J. Kittler, The subspace approach to pattern recognition, in: R. Trappl, G.J. Klir, L. Ricciardi (Eds.), Progress in cybernetics and systems research, Hamisphere Publishing Corporation, 1978, p. 92.

[13] T. Kohonen, Self-Organization and Associative Memory, third ed., Springer, New York, 1989.

[14] J. Laaksonen, E. Oja, Subspace dimension selection and averaged learning subspace method in handwritten digit classification, in: Proceedings of the 6th International Conference on Artificial Neural Networks, 1996, pp. 227-232.

[15] E. Maeda, H. Murase, Kernel based nonlinear subspace method for pattern recognition, Denshi Joho Tsushin Gakkai Ronbunshi D-II J82-D-II 4 (1999) 600-612.

[16] E. Oja, Subspace methods of pattern recognition. Research Studies Press, England, 1983.

[17] R. Radhakrishnan, A.M. Kshirsagar, Influence functions for certain parameters in multivariate analysis, Communications in Statistics A 10 (1981) 515-529. 
[18] Y. Tanaka, Sensitivity analysis in principal component analysis: Influence on the subspace spanned by principal components, Communications in Statistics. A 17-9 (1988) 3157-3175.

[19] Y. Tanaka, Recent advance in sensitivity analysis in multivariate statistical methods, Journal of the Japanese Society of Computational Statistics 7 (1994) 1-25.

[20] S. Watanabe, P.F. Lambert, C.A. Kulikowski, J.L. Buxton, R. Walker, Evaluation and selection of variables in pattern recognition, Computer and Information Sciences II, Academic Press, New York, 1967.

[21] S. Watanabe, Featuring compression, Advances in information systems science Volume 3, in: J.T. Tou (Ed.), Plenum Press, New York, 1970.

[22] S. Watanabe, N. Pakvasa, Subspace method of pattern recognition, in: Proceedings of the 1st International Conference of Pattern Recognition, 1973, pp. 25-32.

[23] Y. Yamashita, T. Wakahara, Subspace methods with globally/locally weighted correlation matrix, in: Proceedings of the 20th International Conference on Pattern Recognition, 2010, pp. 4259-4262. 

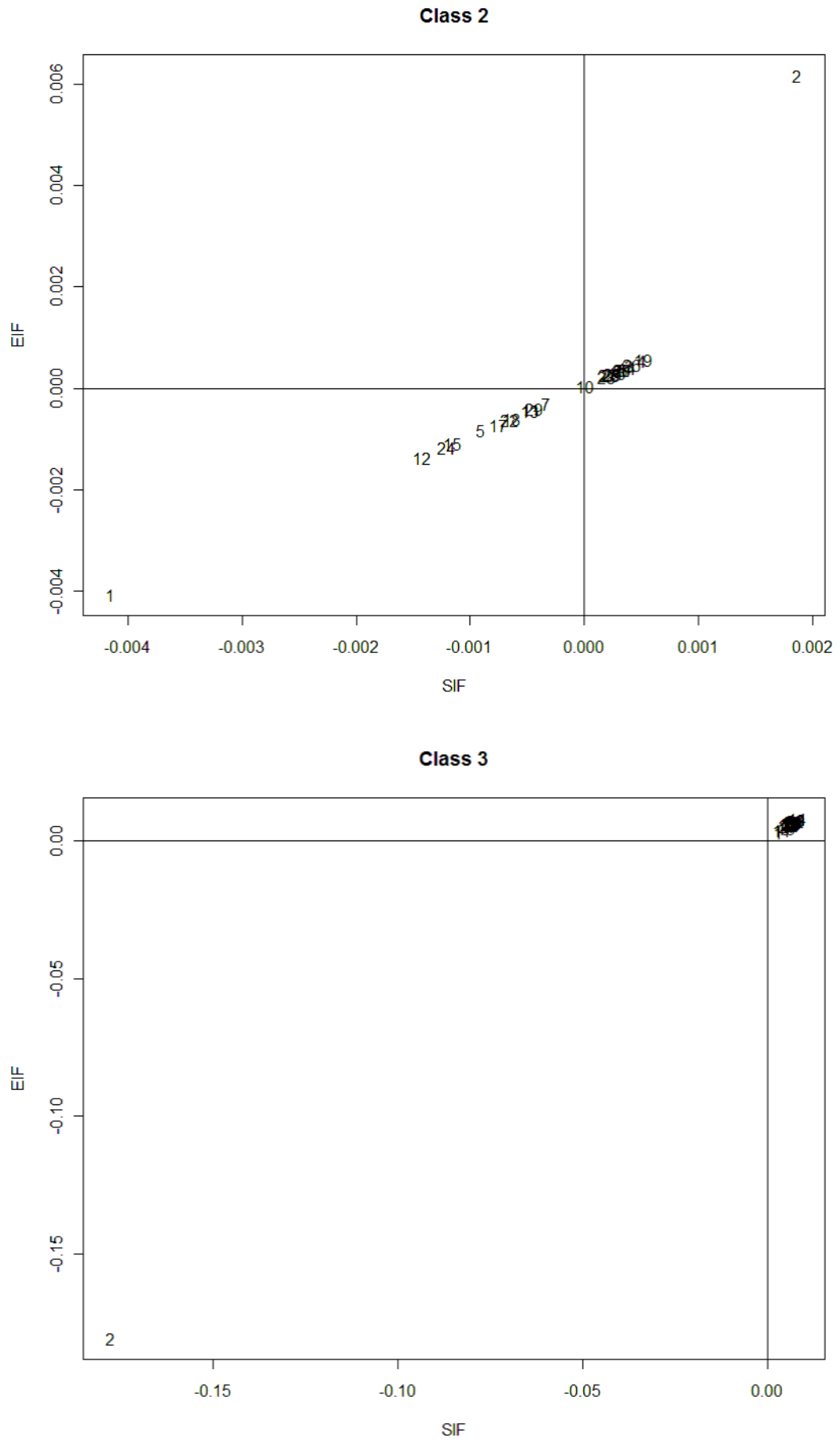

Figure 2: $S I F$ s relative to $E I F$ s by perturbing the training samples in Class 1 

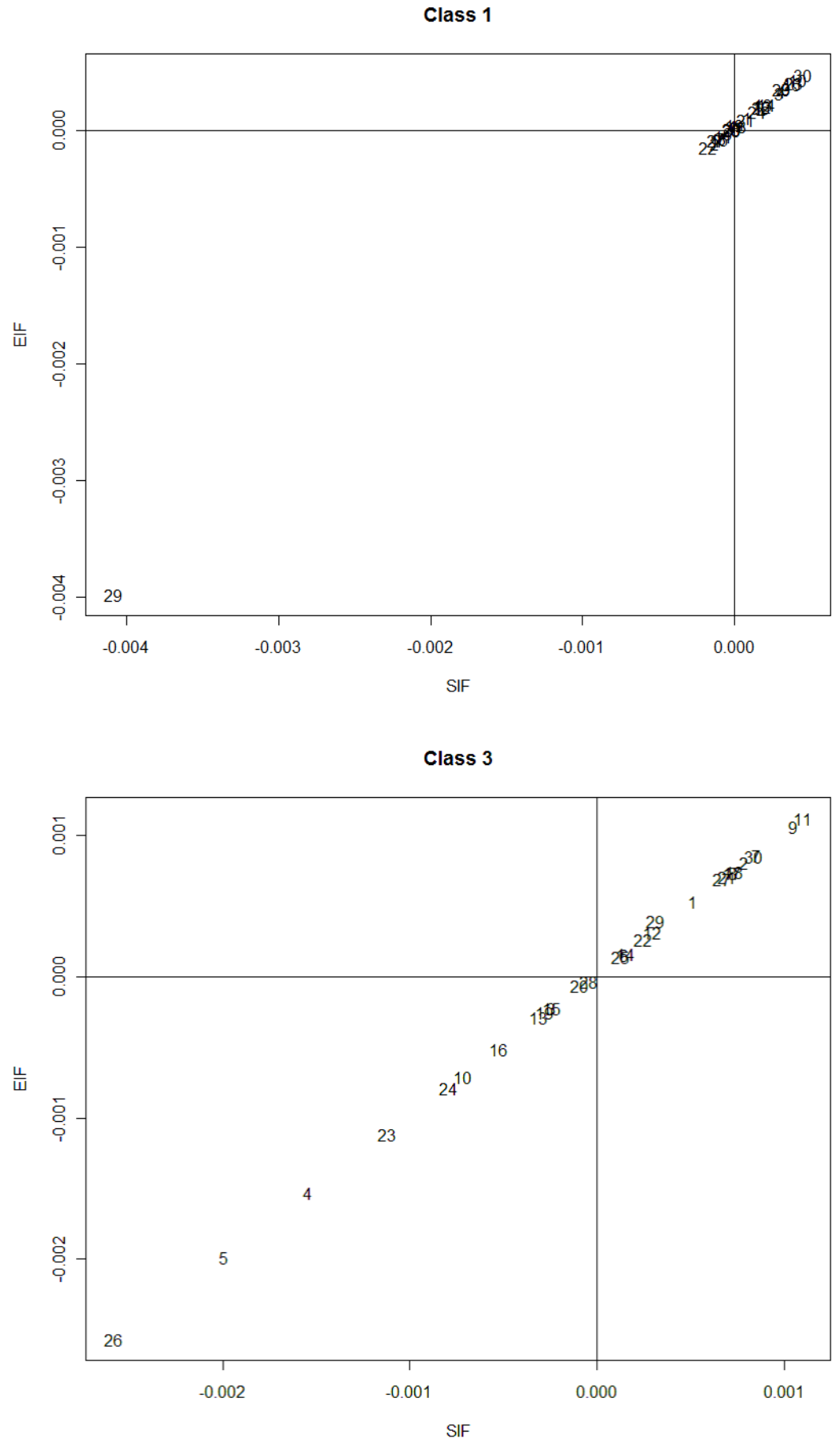

Figure 3: $S I F$ s relative to EIFs by perturbing the training samples in Class 2 

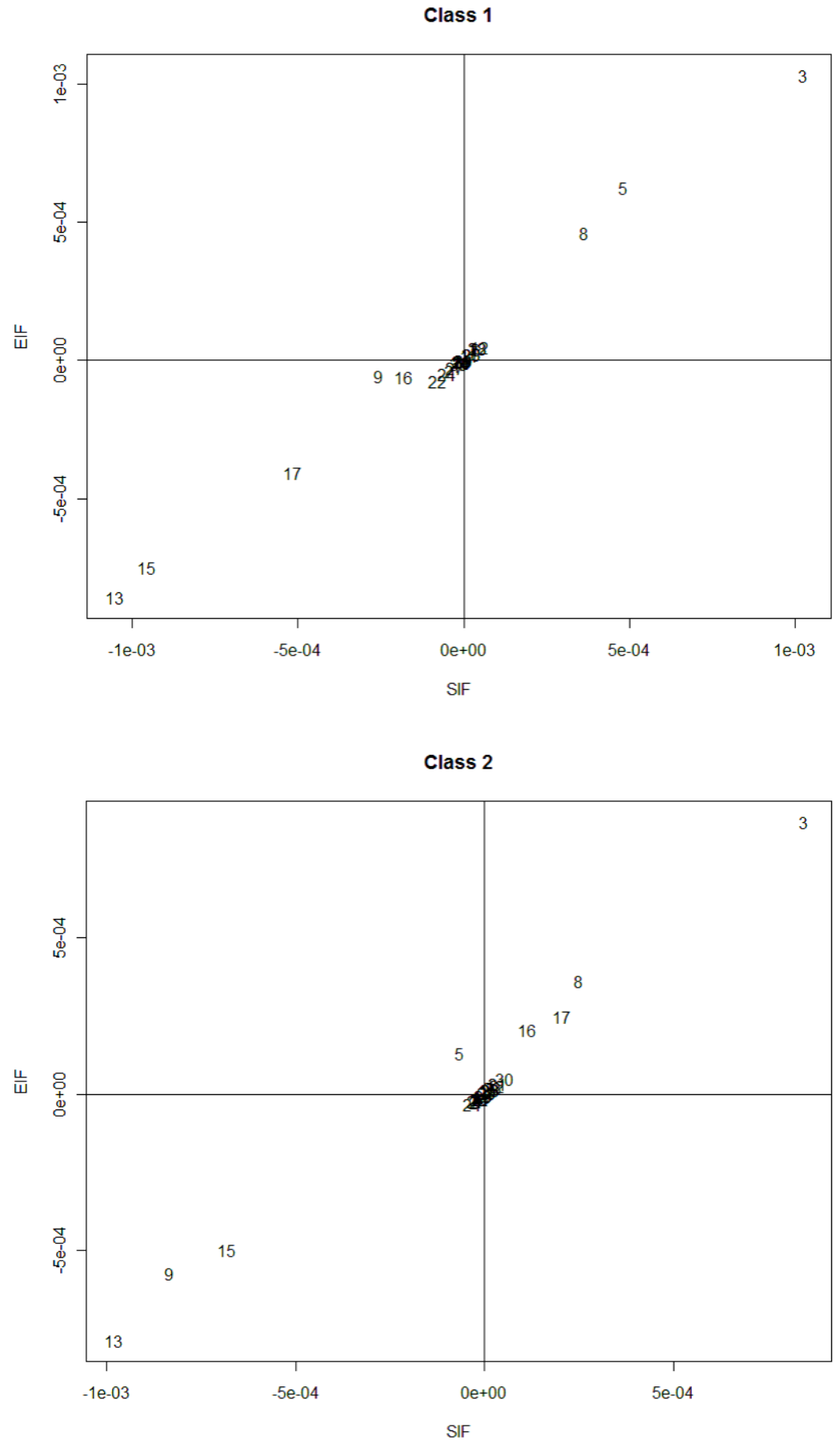

Figure 4: $S I F$ s relative to $E I F$ s by perturbing the training samples in Class 3 


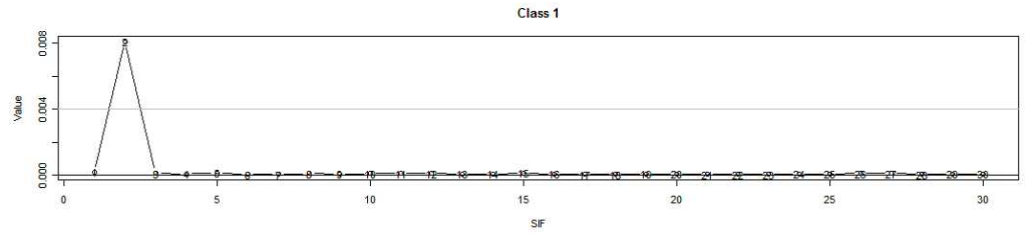

Class 2

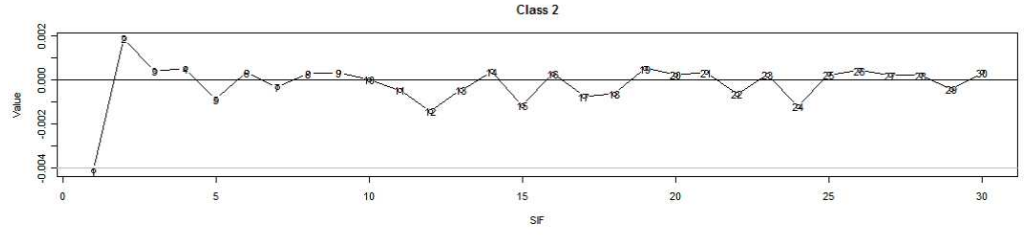

Class 3

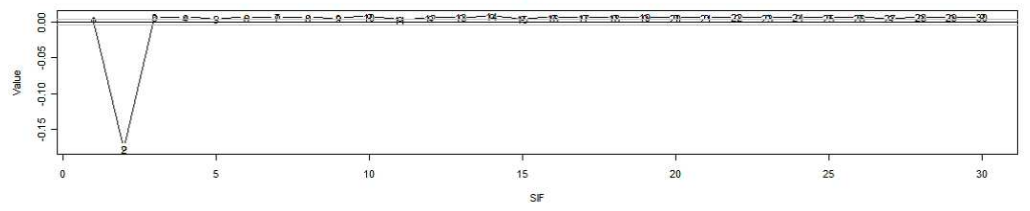

Figure 5: SIFs in each class by deleting the training samples in Class 1
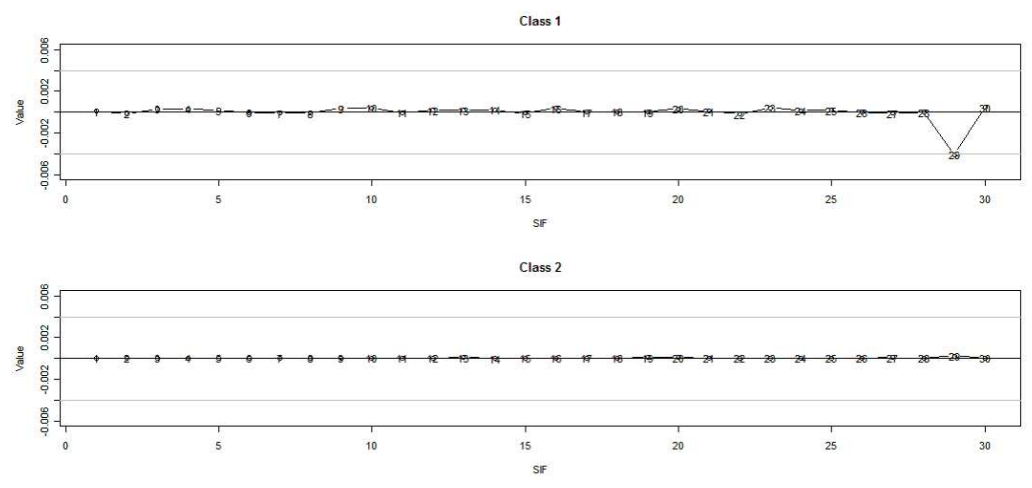

Class 3

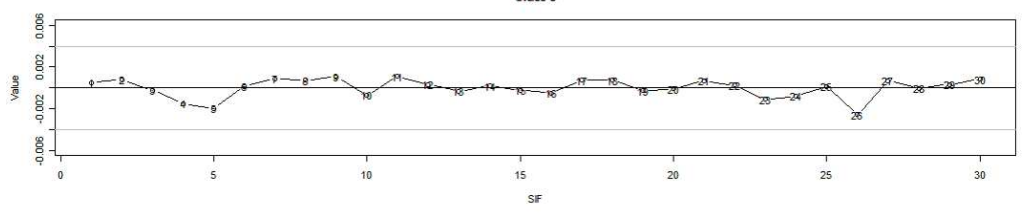

Figure 6: SIFs in each class by deleting the training samples in Class 2 


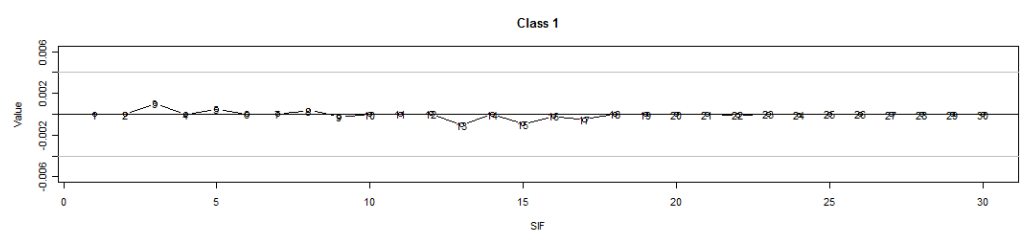

Class 2

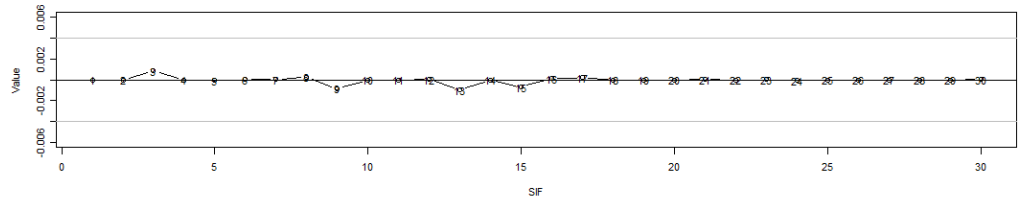

Class 3

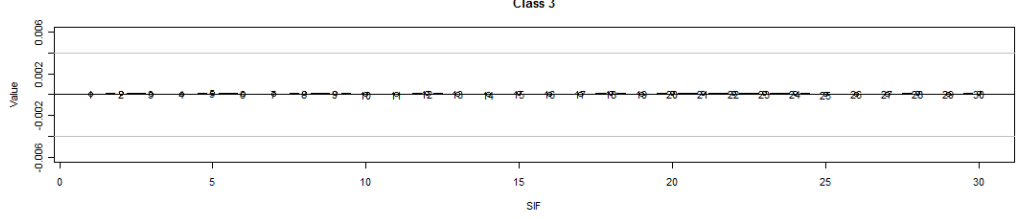

Figure 7: SIFs in each class by deleting the training samples in Class 3

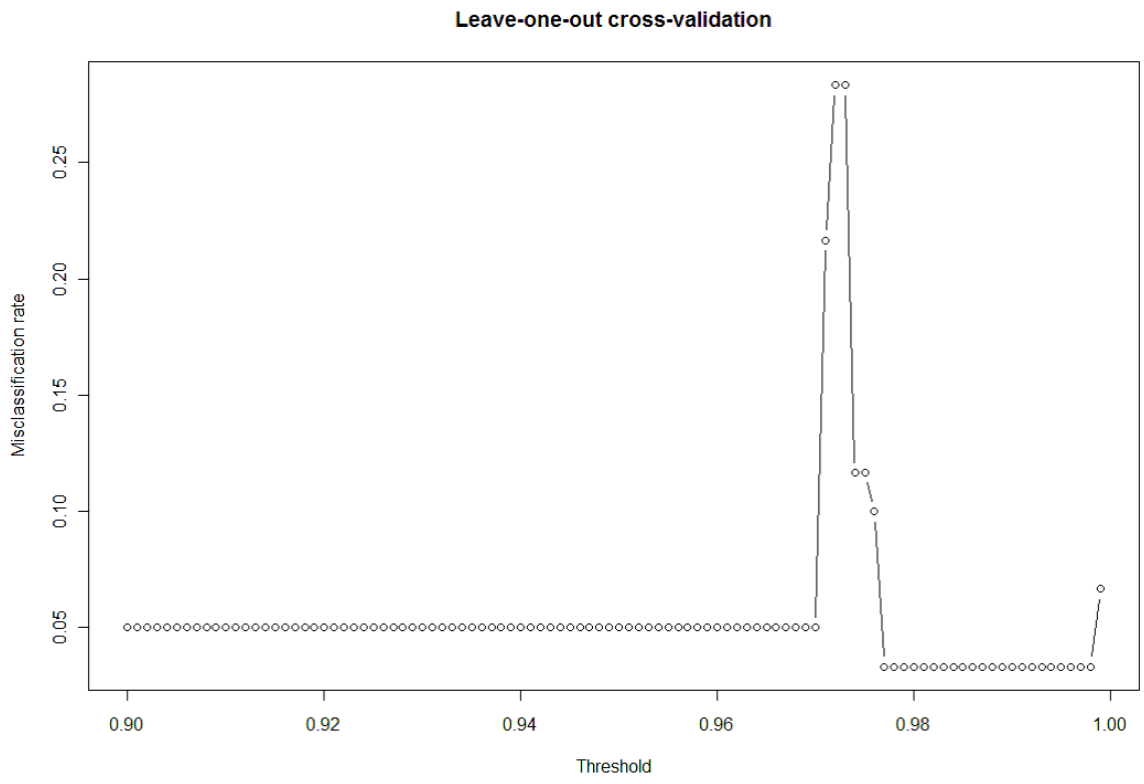

Figure 8: Model selection in CLAFIC 

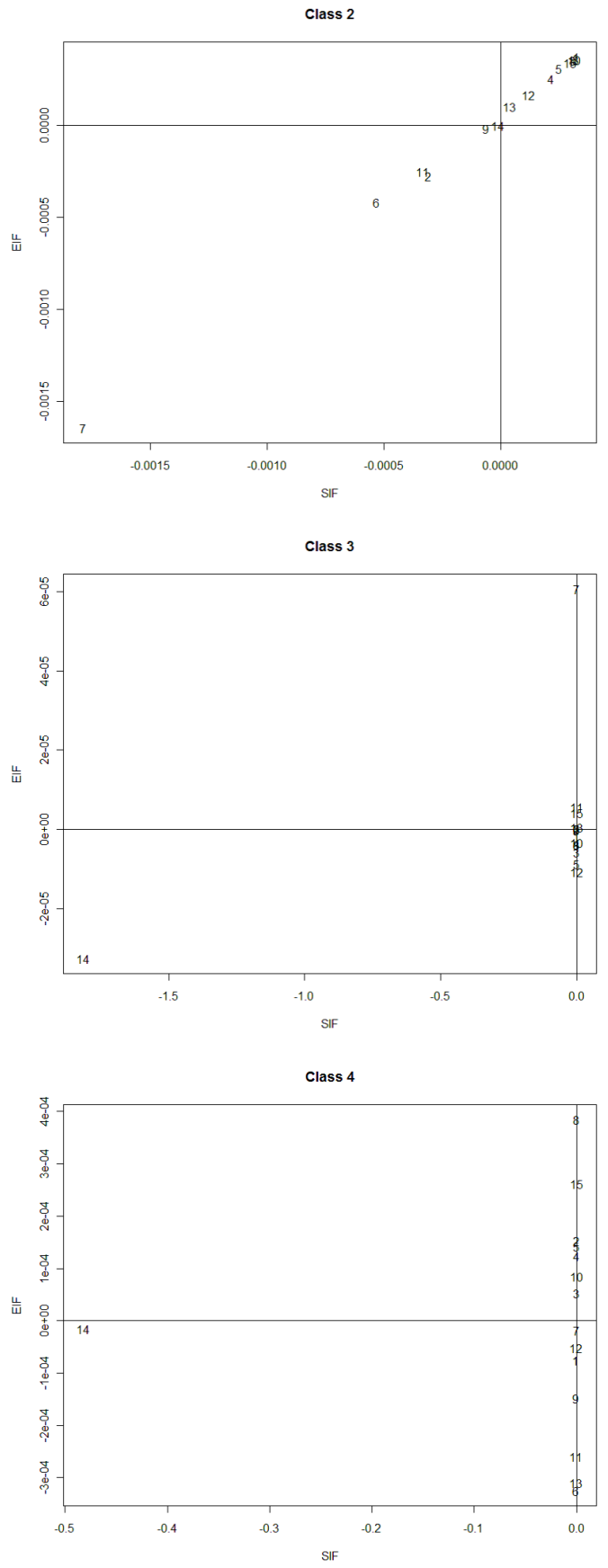

Figure 9: SIFs in each class by deleting the training samples in Class 1 

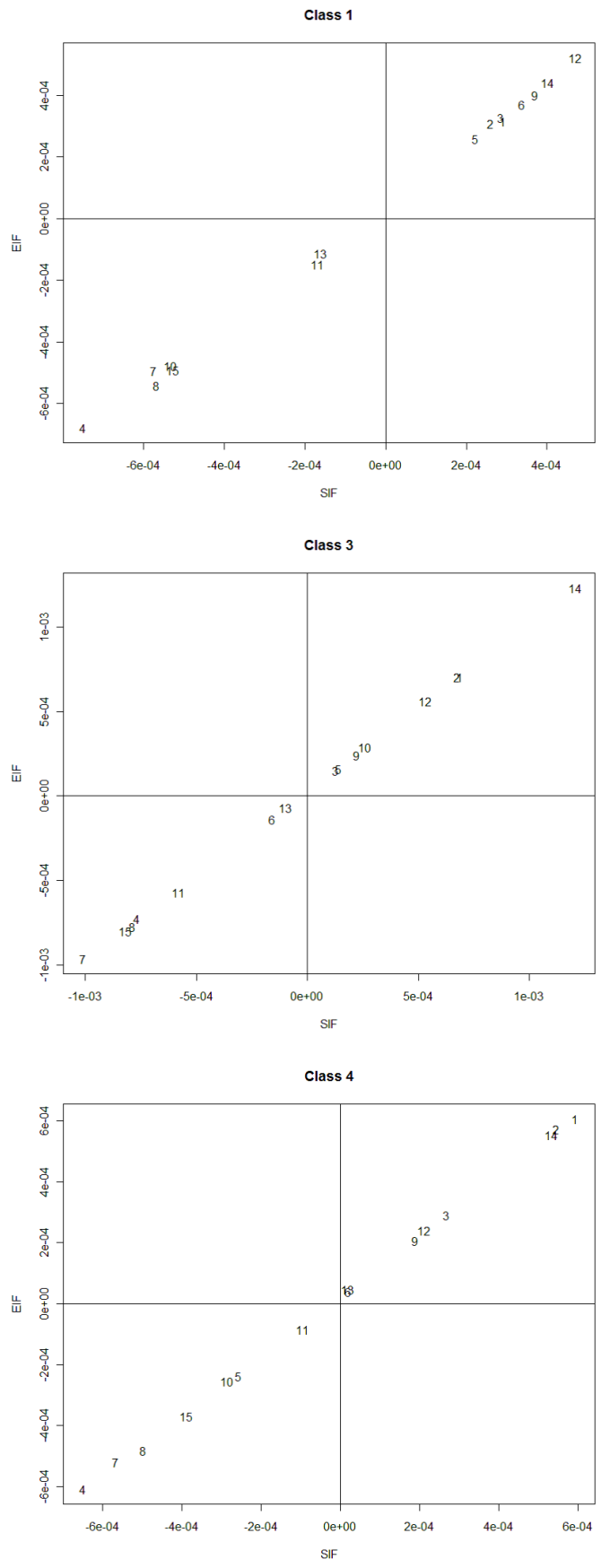

Figure 10: $S I F$ s in each class by deleting the training samples in Class 2 

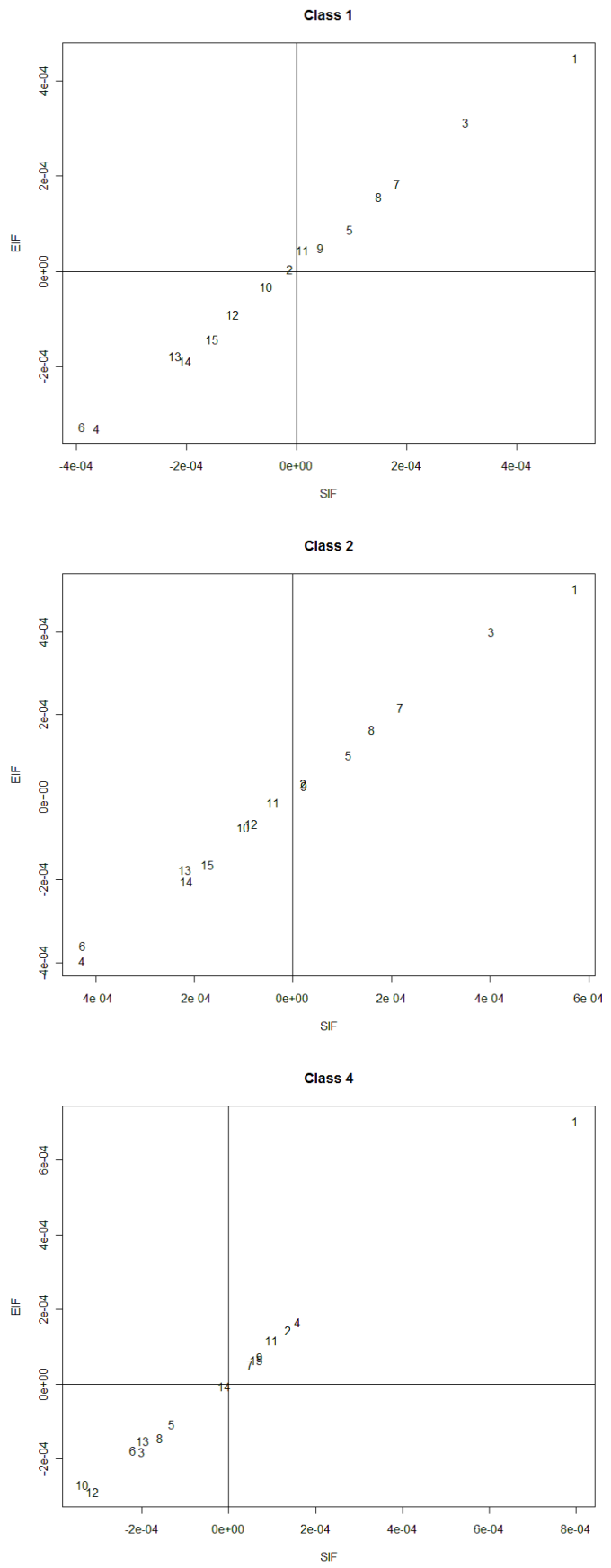

Figure 11: $S I F$ s in each class by deleting the training samples in Class 3 

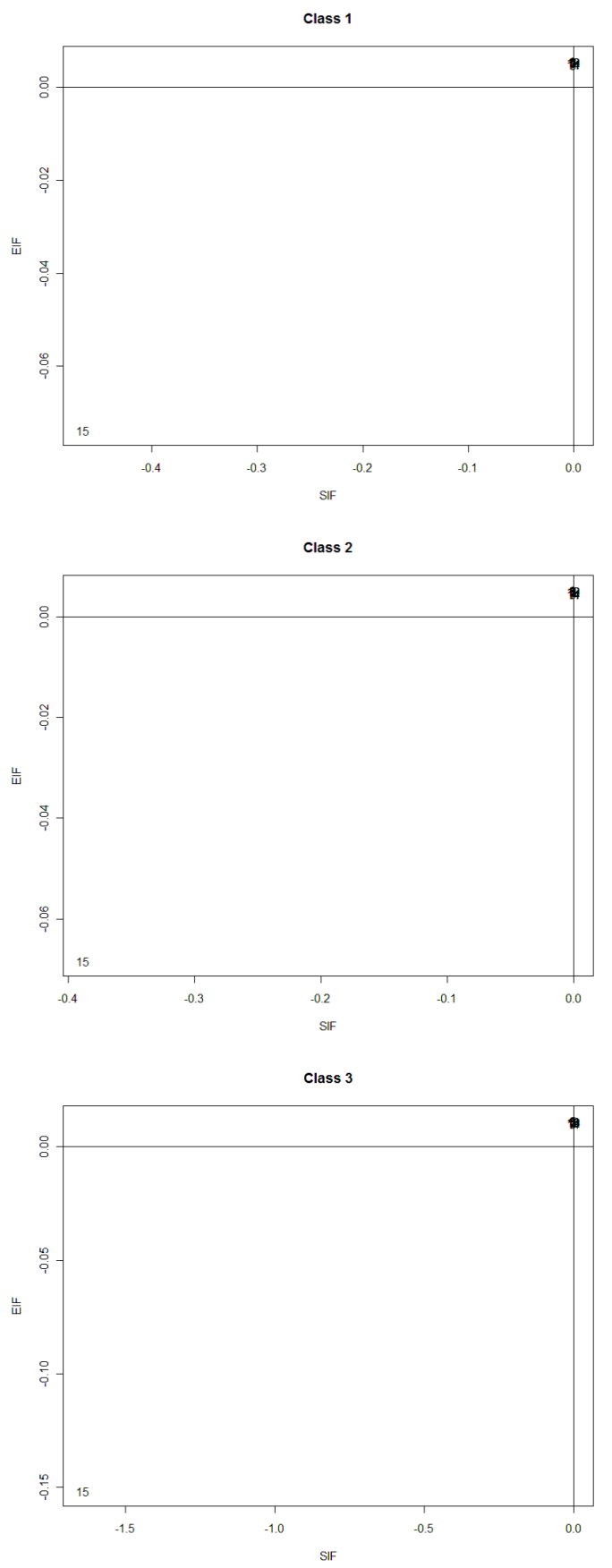

Figure 12: SIFs in each class by deleting the training samples in Class 4 

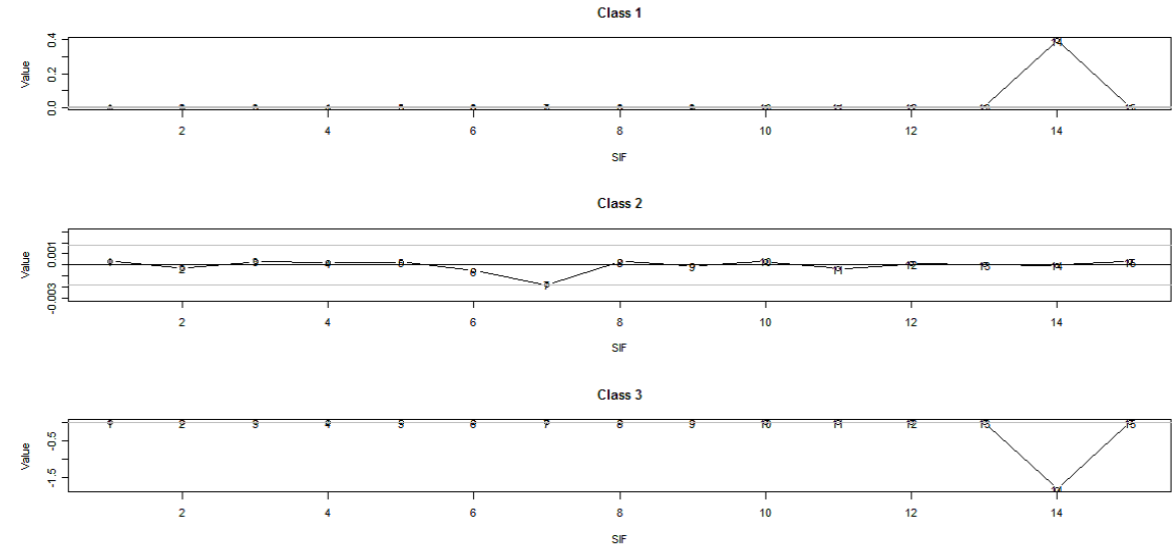

Class 4

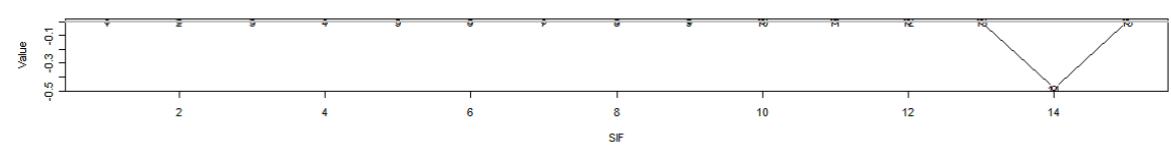

Figure 13: SIFs in each class by deleting the training samples in Class 1

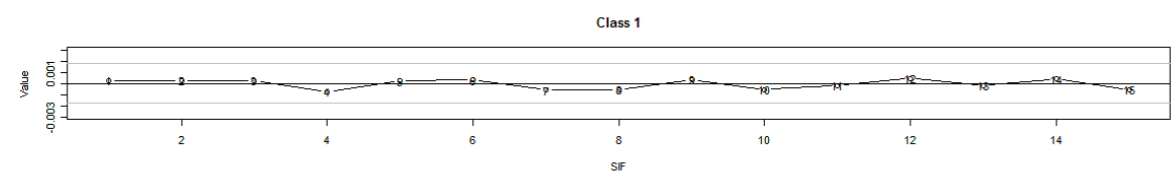

Class 2

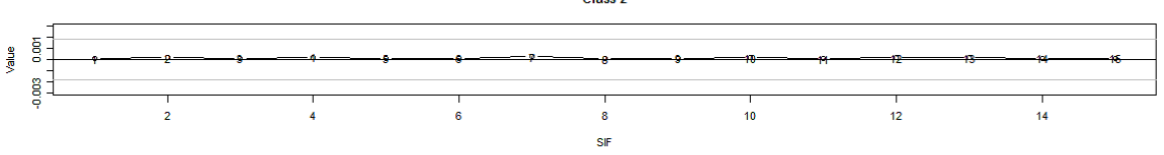

Class 3

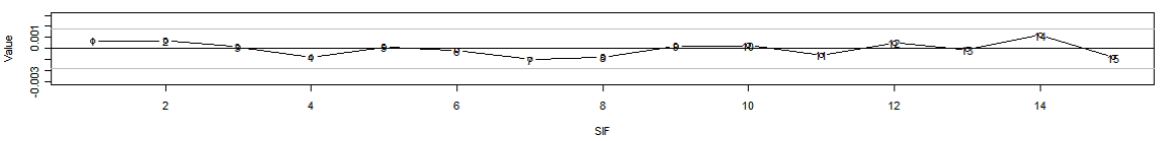

Class 4

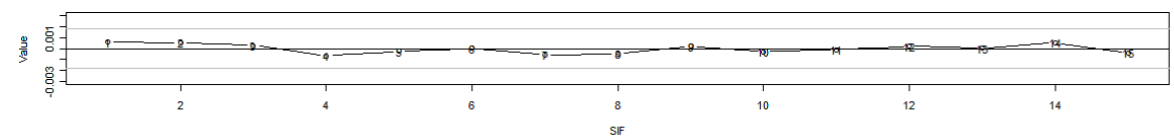

Figure 14: SIFs in each class by deleting the training samples in Class 2 

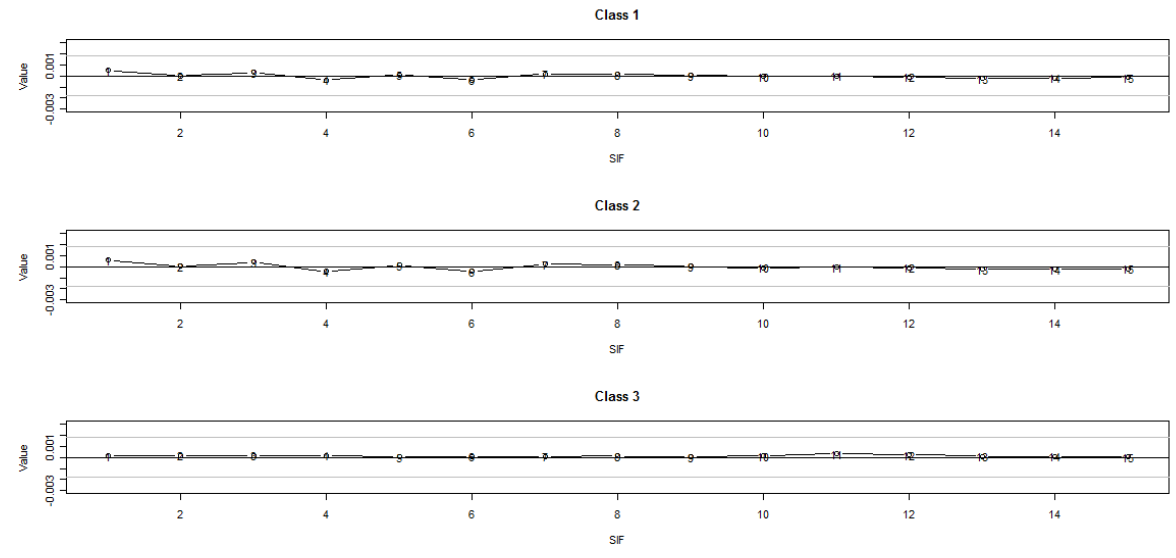

lass 4

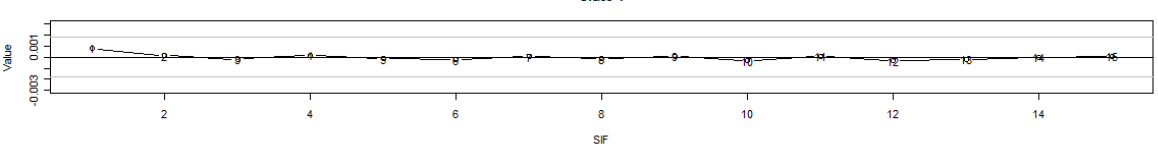

Figure 15: SIFs in each class by deleting the training samples in Class 3
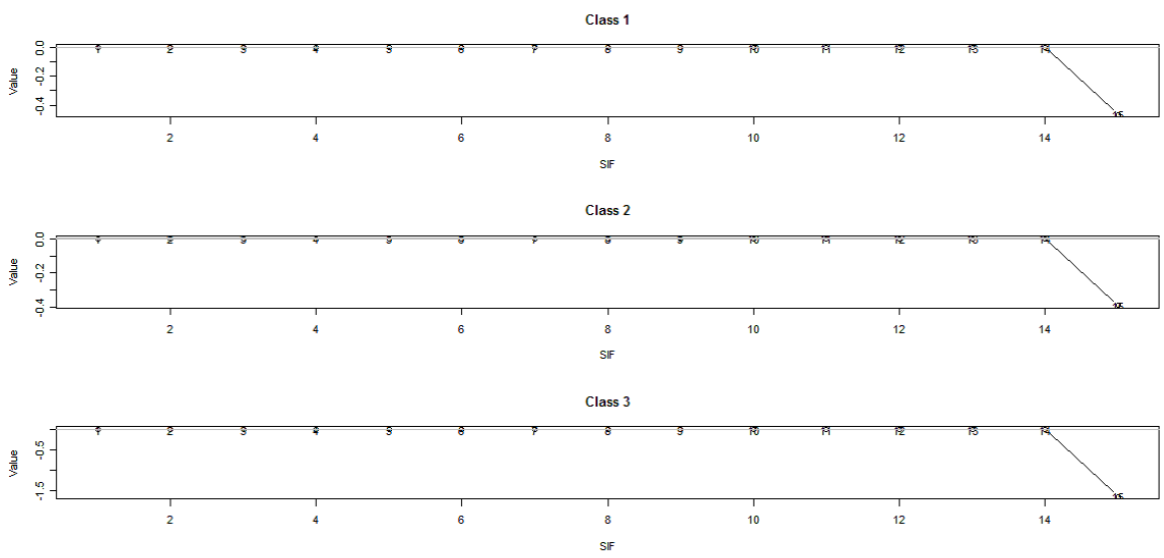

Class 4

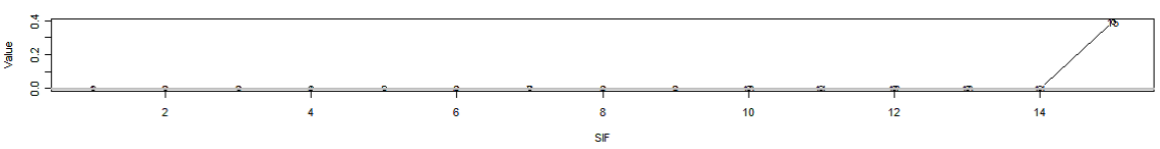

Figure 16: $S I F$ s in each class by deleting the training samples in Class 4 


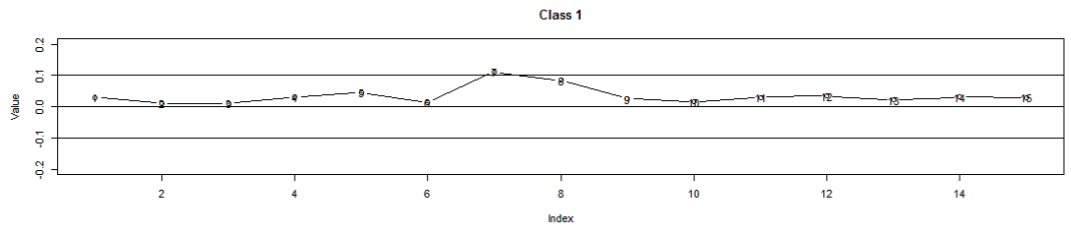

Class 7

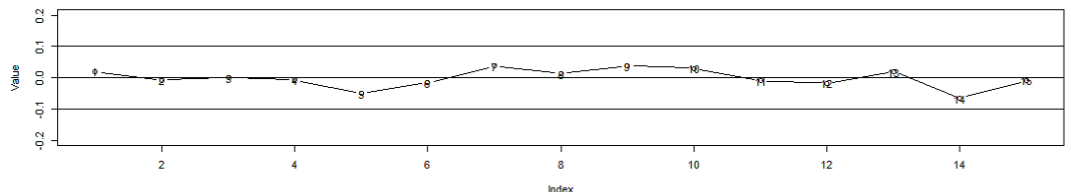

Class 9

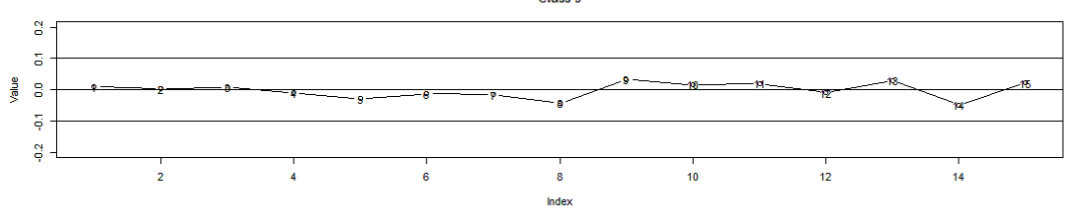

Figure 17: SIFs for each class obtained by deleting training samples in Class "1".

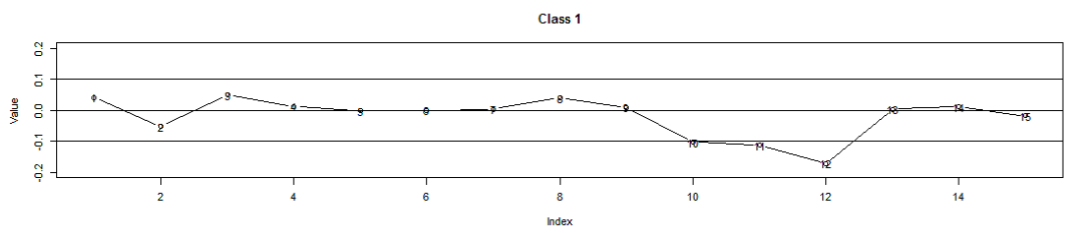

Class 7

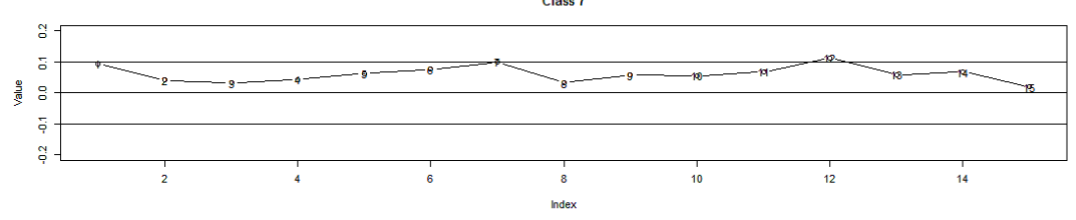

Class 9

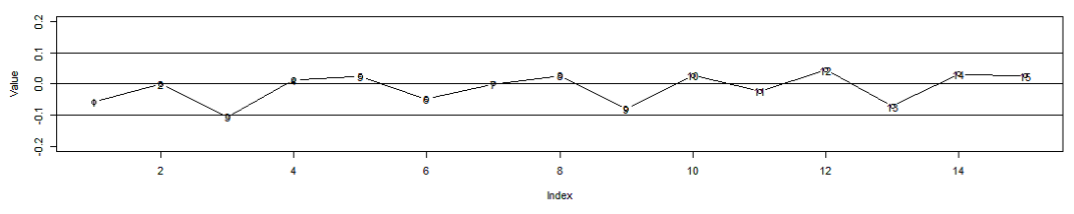

Figure 18: SIFs for each class obtained by deleting training samples in Class "7". 

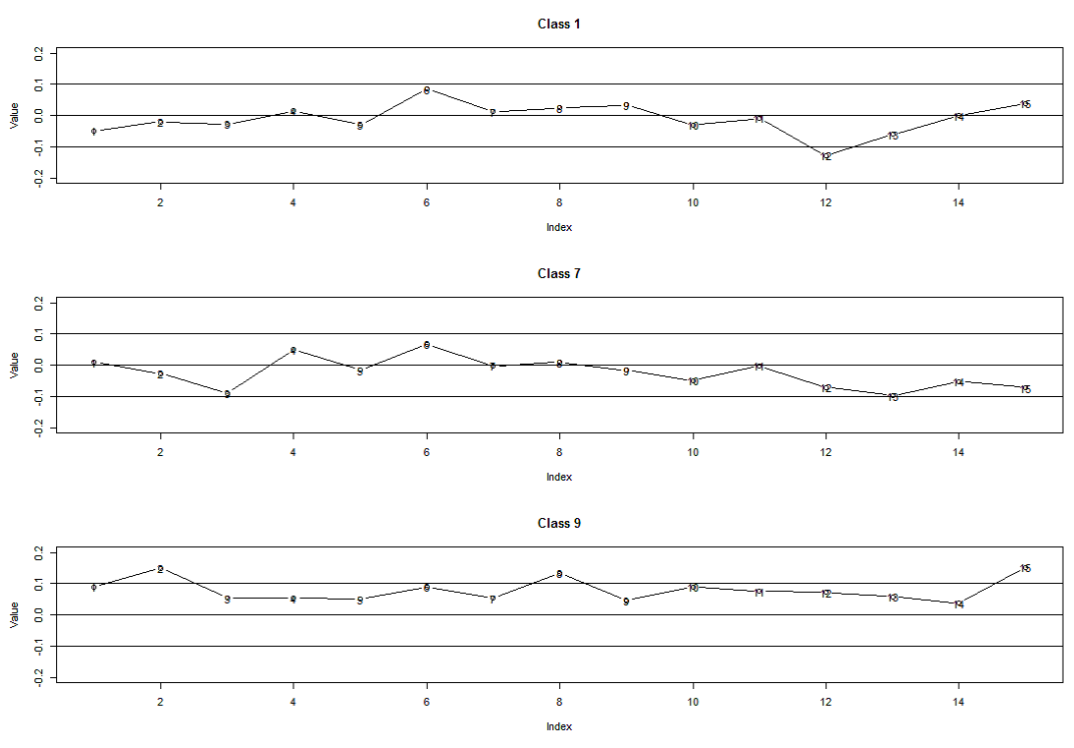

Figure 19: $S I F$ s for each class obtained by deleting training samples in Class "9". 